\title{
Speckle reduction of reconstructions of digital holograms using three dimensional filtering
}

\author{
Jonathan Maycock ${ }^{\mathrm{a}}$, John B. McDonald ${ }^{\mathrm{b}, \mathrm{d}}$, Bryan M. Hennelly ${ }^{\mathrm{c}, \mathrm{d}}$ \\ ${ }^{a}$ Neuroinformatics Group, Department of Information Science, Bielefeld University, \\ Germany \\ ${ }^{b}$ Department of Computer Science, National University of Ireland Maynooth, County \\ Kildare, Ireland \\ ${ }^{c}$ Department of Electronic Engineering, National University of Ireland Maynooth, \\ County Kildare, Ireland \\ ${ }^{d}$ The Callan Institute, National University of Ireland, Maynooth, County Kildare, Ireland
}

\begin{abstract}
We report on a new digital signal processing technique that reduces speckle in reconstructions of digital holograms. This is achieved by convolving the three dimensional intensity pattern (the intensity of the propagated DH at a series of different distances) with a 3D point spread function in all three dimensions $(x, y, z)$. It is based on the fact that the addition of different independent speckle images on an intensity basis reduces the speckle content. We provide quantitative results in terms of speckle index and resolution, and show that filtering in the $z$ direction has the added benefit of an increase the depth of focus of the digital hologram reconstruction.
\end{abstract}

Keywords: Digital Holography, Speckle Reduction, 3D Filtering, Digital signal processing

\section{Introduction}

Digital holography $[1,2,3]$ differs from holography in that an electronic sensor, rather than photographic material, is used to capture a hologram during the recording step. In addition the reconstructions are performed

Email addresses: jmaycock@techfak.uni-bielefeld.de (Jonathan Maycock), johnmcd@cs.nuim.ie (John B. McDonald), Bryan. Hennelly@nuim.ie (Bryan M. Hennelly) 
numerically on a computer instead of physically reconstructing the hologram. In 1994, Schnars and Jüptner [3] were the first to successfully capture Fresnel holograms with a CCD sensor. These digital holograms, like their analogue counterparts, require light that has a high level of coherence and therefore suffer from the effects of speckle noise.

Speckle occurs when coherent light is incident on an optically rough surface [4]. It degrades the quality of the resulting images and makes it difficult or impossible to resolve fine detail. All coherent imaging systems suffer from speckle and when we consider viewing reconstructions of DHs this speckle is an unwanted noise [5]. The presence of speckle hinders the application of image processing techniques to digital holographic data. The reduction of speckle is therefore an important topic whenever reconstructions of DHs are to be used. One such area which benefits from speckle reduction is the extraction of 3D shape information from DHs. Existing focus measures $[6,7,8,9,10]$ are hampered by the speckled appearance of the reconstructions, and results are improved when a pre-processing speckle reduction step is used. Another area that benefits from speckle reduced DH reconstructions is that of pattern recognition $[11,12,13,14]$. The analysis of particles for flow field measurement using reconstructions of DHs has also benefitted from speckle reduction [15]. However, some of the aforementioned papers use standard noise reduction techniques that have not been developed and optimized to address the underlying properties of speckle in DHs and therefore produce results that are not optimal.

There have been a number of recent contributions to the area of speckle reduction in DH reconstructions. In 2004, Dubois et al. [15] used a spatially partially coherent source during recording to reduce the effects of speckle in a digital holographic microscopy system. In order to create the spatially partially coherent source a rotating diffuser was placed in the path of the coherent light source. This technique was also used by Kim [16] in 2004 to reduce speckle in holographic 3D displays and by Sucerquia et al. [17] two years later who introduced static (but different) diffusers during the recording of DHs and then added together the resulting reconstructions. Also in 2006, Baumbach et al. [18] added together reconstructions from laterally shifted holograms of an object to reduce the speckle content. Nomura et al. [19] proposed reducing speckle in reconstructions of DHs by superposing reconstructed DHs recorded at different wavelengths. Using a wavelengthtunable laser, they added together reconstructions from each of the captured DHs on an intensity basis, thus reducing the speckle content. The imple- 
mentation of a synthetic aperture for digital holography has been realised recently $[20,21,22,23,24,25]$. It is achieved by moving either the CCD or the sample, capturing multiple DHs between successive moves, and then stitching together the resulting DHs to form a larger DH. The primary purpose of a synthetic aperture is to increase the resolution of the resulting images, while at the same time increasing the aperture size causes the average speckle size to reduce. All of these techniques required a change to the setup (i.e., the introduction of diffusers) and/or multiple captures of holograms to achieve a reduction in speckle.

There have also been contributions to speckle reduction in which only a single DH was used. To lessen the effects of speckle noise, while investigating compression and Internet transmission of DHs, Naughton et al. [26] applied a subsampling (spatial integration) operation. They applied this to the intensity in the reconstruction plane where the operation integrates nonoverlapping blocks of $n \times n$ pixels to a single value. Garcia-Sucerquia et al. [27] also used a subsampling operation to reduce the effects of speckle in reconstructions of DHs. Furthermore, they suggested the merging of the subsampling operation with that of the median filter, although no quantative results were given in terms of the effects on resolution. In 2004, Bertaux et al. [28], put forward an approach for the removal of speckle, which was based on the maximum-likelihood technique and used a general model for image reflectivity. The image was further improved by applying a constraint on isoline gray levels, which results in smoothness without blurring of the edges of the objects in the image. Finally, in 2006 Charrire et al. [29] convolved reconstructions with a Gaussian filter to reduce noise in images of cells captured by a DHM system. However as the focus of their paper was not on speckle reduction, but on living specimen tomography, details of the filtering process they used was not given.

We report on a new digital signal processing technique that reduces speckle in reconstructions of digital holograms (DHs). This is achieved by convolving the three dimensional (3D) intensity pattern (the intensity of the propagated $\mathrm{DH}$ at a series of different distances) with a $3 \mathrm{D}$ point spread function in all three dimensions $(x, y, z)$. This 3D filtering method is based on the fact that the addition of different independent speckle images on an intensity basis reduces the speckle content, and we must therefore take into account the size of the speckle in all three dimensions. In contrast to some of the approaches outlined above, our technique requires just a single $\mathrm{DH}$ and unlike standard image processing noise reduction techniques uses the 
inherent properties of the speckle field to adjust the size of the filter used.

We begin by investigating the two dimensional (2D) case where different speckle patterns are produced by laterally shifting the $\mathrm{DH}$ reconstruction plane in $(x, y)$. If the shift is greater than a speckle correlation area (average speckle size), then the resulting speckle patterns will be independent and when summed on an intensity basis, this results in speckle reduction. Adding together a number of shifted copies of the 2D intensities, each differently weighted in the sum, may be described by a $2 \mathrm{D}$ convolution. Convolution provides a framework for the application of different kinds of filters, e.g., the Gaussian filter. Convolution also provides a framework for extension to the case of 3D filtering. In this case we reconstruct a stack of intensities at a range of different distances, separated by a constant sampling interval, $T_{z}$, in the $z$ direction. If the sampling distance along $z$ is at least as large as the speckle size in the $z$ direction we may assume that each reconstruction has an independent speckle pattern. While applying our method we must also consider its impact in terms of blurring the image that we are seeking to improve.

The idea of adding shifted intensities for speckle reduction in holography has precedent. In 1975, Gama [30] used a vibrating source during optical replay of holographic reconstructions of diffuse objects to reduce the speckle contrast pattern. Recently, Pen et al. [31] introduced a method to reduce coherent noise in digital holographic phase contrast microscopy by slightly shifting the specimen under investigation and at each shift capturing a DH, each with a different speckle pattern. By adding reconstructions from these DHs together the speckle content was reduced. Another method by the same group introduced an improved polarization recording approach to reduce speckle noise in off-axis DHs [32]. Once again multiple DHs are obtained by rotating the linear polarization state of both illumination and reference beams simultaneously. Speckle is suppressed by adding together reconstructions from these DHs on an intensity basis. Nomura et al. [19] proposed a different method of reducing speckle by superposing the intensities of reconstructed DHs recorded at different wavelengths. They used a wavelength-tunable laser and they captured their DHs in the range $567 \mathrm{~nm}$ to $624 \mathrm{~nm}$, with an interval of $8 \mathrm{~nm}$. They then added together reconstructions from each of the captured DHs on an intensity basis, thus reducing the speckle content. While these final two approaches do not compromise the underlying image quality, they have the major drawback that multiple DHs need to be captured, ruling out the possibility of investigating dynamic 
objects.

This paper is structured as follows. Sect. 2 provides a full analysis of our technique and is followed by Sect. 3 which discusses the metrics used to evaluate its effectiveness and provides an analysis of the expected theoretical resolution. Sect. 4 provides a comprehensive set of experimental results for filtering in one, two and three dimensions. Finally we summarize our contribution in Sect. 5.

\section{Analysis}

An analysis of the technique is now presented. In Sect. 2.1 the convolution framework, upon which our technique is built, is discussed. Then we address the issue of generating a 3D reconstructed $\mathrm{DH}$ along with the required sampling intervals needed for convolving such a signal (see Sect. 2.2). The differences between reconstruction with the direct and spectral methods and possible implications for our approach are treated in Sect. 2.3. In Sect. 2.4 we discuss speckle size in 3 dimensions and compare the expected size given by theory to the size of the speckle observed in numerically reconstructed DHs. Finally, the model we have developed is presented in Sect. 2.5.

\subsection{Convolution and filtering}

Convolution in 2D is often used in image processing to reduce noise. However, as we are using DHs, it is possible to create a $3 \mathrm{D}$ signal by calculating the intensity pattern for a stack of different distances within a certain range around the correct object distance. For such a signal we can perform 3D filtering using 3D convolution with a suitable 3D kernel. Following this, the 2D image corresponding to the correct distance can be isolated. 3D convolution in the continuous case can be written as

$$
(g * f)(x, y, z)=\int_{-\infty}^{\infty} \int_{-\infty}^{\infty} \int_{-\infty}^{\infty} f(u, v, w) g(x-u, y-v, z-w) d u d v d w
$$

where $x, y, z, u, v, w$ are $\in \mathbb{R}$ and the discrete bounded case can be formulated as 


\begin{tabular}{|c|c|c|c|c|}
\hline & $T_{x}$ & $T_{y}$ & $T_{z}$ \\
\hline Di. & $\frac{z_{0} \lambda}{N_{x} \Delta \xi}$ & $\frac{z_{0} \lambda}{N_{y} \Delta \eta}$ & $z_{0}\left(\frac{1}{1+\frac{z_{0} \lambda}{\min \left(N_{x}, N_{y}\right)^{2} \min (\Delta \xi, \Delta \eta)^{2}}}\right)-z_{0}\left(\frac{1}{1-\frac{z_{0} \lambda}{\min \left(N_{x}, N_{y}\right)^{2} \min (\Delta \xi, \Delta \eta)^{2}}}\right) \mid$ \\
Sp. & $\Delta \xi$ & $\Delta \eta$ & $\left|z_{0}\left(\frac{\min \left(N_{x}, N_{y}\right)}{\min \left(N_{x}, N_{y}\right)+1}\right)-z_{0}\left(\frac{\min \left(N_{x}, N_{y}\right)}{\min \left(N_{x}, N_{y}\right)-1}\right)\right|$ \\
\hline
\end{tabular}

Table 1: Sampling intervals for the 3D field when using the direct method and the spectral method for simulating the Fresnel Transform. Di.: Direct method of reconstruction, Sp.: Spectral method of reconstruction, $T_{x}$ : Sampling interval in $x, T_{y}$ : Sampling interval in $y$, $T_{z}$ : Sampling interval in $z$ (depth of focus [5]), $\Delta \xi$ : sensor pixel size in $x, \Delta \eta$ : sensor pixel size in $y, N_{x}, N_{y}$ : number of pixels on the sensor in $x$ and $y$ respectively, $z_{0}$ : distance of the object to the sensor, $\lambda$ : wavelength of the light used.

$$
\begin{aligned}
h\left[r^{\prime} T_{x}, s^{\prime} T_{y}, t^{\prime} T_{z}\right] & =\sum_{r=-\frac{N x}{2}}^{\frac{N x}{2}} \sum_{s=-\frac{N y}{2}}^{\frac{N y}{2}} \sum_{t=-\frac{N z}{2}}^{\frac{N z}{2}} f\left[r T_{x}, s T_{y}, t T_{z}\right] \\
& \times g\left[r^{\prime} T_{x}-r T_{x}, s^{\prime} T_{y}-s T_{y}, t^{\prime} T_{z}-t T_{z}\right]
\end{aligned}
$$

where $f$ is the $3 \mathrm{D}$ signal, $g$ the $3 \mathrm{D}$ kernel, $T_{x}, T_{y}$ and $T_{z}$ the sampling periods in the $(x, y, z)$ directions respectively and $r, s, t, r^{\prime}, s^{\prime}$ and $t^{\prime}$ are integers with a range of $-\frac{N x}{2}$ to $\frac{N x}{2},-\frac{N y}{2}$ to $\frac{N y}{2}$ and $-\frac{N z}{2}$ to $\frac{N z}{2}$, respectively. Rather than using a simple block filter, we can use on a kernel based on important image feature directions, use a Gaussian filter, or other image processing filters such as a Laplacian filter.

\subsection{Numerical computation of the $3 D$ intensity field}

Discrete convolution with a 3D filter kernel requires a 3D discrete signal. When constructing such a signal, careful consideration needs to be focused on the discrete sampling intervals between the $2 \mathrm{D}$ slices in the $z$ direction. Furthermore, the sampling intervals differ depending on the choice of reconstruction method. Table 1 gives the equations used to calculate sampling intervals in the $x, y$ and $z$ directions [5]. As can be seen from the table, the sampling interval in $x$ and $y\left(T_{x}\right.$ and $\left.T_{y}\right)$ is proportional to the reconstruction distance when using the direct method and is the same size as the sensor pixels when using the spectral method. In order to decide which sampling interval we should choose along the $z$ direction, we use the concept of depth of focus given by Kreis [5]. Kreis calculates the depth of focus to be related 
to the rate of change along the $z$ direction and therefore the correct sampling interval in the Nyquist sense. In order to assure sufficient sampling, the 3D intensity signal should be made up of a number of $2 \mathrm{D}$ reconstructions (zero-padded sufficiently) that are centered on the optimum reconstruction distance and are separated in space by $T_{z}$. We further reduced this sampling interval to a quarter of its size to ensure sufficient sampling of the 3D intensity field. The effect of this is to increase computation times by a factor of 4 , but this was acceptable for our set of tests as these intensities only had to be performed once, and could be stored for further processing.

\subsection{Direct and spectral methods of reconstruction}

The direct method of reconstruction [33] can be derived by applying the Fresnel Transform to the sampled signal $O\left(n_{x} \Delta \xi, n_{y} \Delta \eta\right)$, which denotes the sampled object term in the hologram (CCD) plane to obtain an equation for the continuous reconstructed image $\operatorname{recon}\left(x^{\prime}, y^{\prime}\right)$. It is given by

$$
\begin{aligned}
\operatorname{recon}\left(x^{\prime}, y^{\prime}\right) & =\frac{\exp [i k d]}{j \lambda z_{0}} \exp \left[\frac{j \pi}{z_{0} \lambda}\left(x^{\prime 2}+y^{\prime 2}\right)\right] \\
& \times \iint O\left(n_{x} \Delta \xi, n_{y} \Delta \eta\right) \exp \left[\frac{j \pi}{z_{0} \lambda}\left(x^{2}+y^{2}\right)\right] \\
& \times \exp \left[\frac{j 2 \pi}{\lambda z_{0}}\left(x^{\prime}+y^{\prime}\right)\right] \mathrm{d} x \mathrm{~d} y .
\end{aligned}
$$

The constant $\frac{\exp [j k d]}{j \lambda d}$ does not depend on the spatial frequency coordinates or the object and so shall be omitted. We note that since the signal $\mathrm{O}$ exists only at discrete spatial coordinates, only these discrete values of $x$ and $y$ need to be considered in the above integral;

$$
\begin{array}{ll}
x=n_{x} \Delta \xi & n_{x}=-\frac{N_{x}}{2}, \ldots ., \frac{N_{x}}{2} \\
y=n_{y} \Delta \eta & n_{y}=-\frac{N_{y}}{2}, \ldots ., \frac{N_{y}}{2},
\end{array}
$$

where $\Delta \xi$ and $\Delta \eta$ are the CCD pixel sizes in the $x$ and $y$ directions respectively, and where $N_{x}$ and $N_{y}$ are the number of pixels in the CCD camera in the $x$ and $y$ directions, respectively. We can conclude that $W_{C C D x}$ and 
$W_{C C D y}$, the widths of the camera in the $x$ and $y$ directions are given by $N_{x} \Delta \xi$ and $N_{y} \Delta \eta$.

If we sample the reconstructed complex image with sampling rate $\Delta \xi^{\prime}$ and $\Delta \eta^{\prime}$ in the reconstruction plane and interest ourselves only in the samples over the range of indices given by

$$
\begin{array}{ll}
x^{\prime}=m_{x^{\prime}} \Delta \xi^{\prime} & m_{x^{\prime}}=-\frac{N_{x}}{2}, \ldots ., \frac{N_{x}}{2}-1 \\
y^{\prime}=m_{y^{\prime}} \Delta \eta^{\prime} & m_{y^{\prime}}=-\frac{N_{y}}{2}, \ldots ., \frac{N_{y}}{2}-1,
\end{array}
$$

and choose the output sampling intervals to be defined as follows

$$
\begin{aligned}
& \Delta \xi^{\prime}=\frac{\lambda z_{0}}{N_{x} \Delta \xi}=\frac{\lambda z_{0}}{W_{C C D x}} \\
& \Delta \eta^{\prime}=\frac{\lambda z_{0}}{N_{y} \Delta \eta}=\frac{\lambda z_{0}}{W_{C C D y}},
\end{aligned}
$$

then Eq. 4 will reduce to the following;

$$
\begin{aligned}
\operatorname{recon}\left(m_{x^{\prime}} \Delta \xi^{\prime}, m_{y^{\prime}} \Delta \eta^{\prime}\right) & =\exp \left[\frac{j \pi}{z_{0} \lambda}\left(\left(\lambda z_{0} m_{x^{\prime}} \Delta \xi^{\prime}\right)^{2}+\left(\lambda z_{0} m_{y^{\prime}} \Delta \eta^{\prime}\right)^{2}\right)\right] \\
& \times \sum_{n_{x}=\frac{N_{x}}{2}}^{\frac{N_{x}}{2}-1} \sum_{n_{y}=\frac{N_{y}}{2}}^{\frac{N_{y}}{2}-1} O\left(n_{x} \Delta \xi, n_{x} \Delta \eta\right) \exp \left[\frac{j \pi}{z_{0} \lambda}\left(\left(n_{x} \Delta \xi\right)^{2}+\left(n_{y} \Delta \eta\right)^{2}\right)\right] \\
& \times \exp \left[j 2 \pi \frac{n_{x} m_{x^{\prime}}}{N_{x}}+j 2 \pi \frac{n_{y} m_{y^{\prime}}}{N_{y}}\right]
\end{aligned}
$$

which is in the form of a Discrete Fourier Transform (DFT). This equation can be calculated using the very time efficient Fast Fourier Transform (FFT) algorithm. We note that the output window size in the $x^{\prime}$ and $y^{\prime}$ dimensions can be calculated to be

$$
\begin{aligned}
& N_{x} \Delta \xi^{\prime}=\frac{\lambda z_{0}}{\Delta \xi}, \\
& N_{y} \Delta \eta^{\prime}=\frac{\lambda z_{0}}{\Delta \eta} .
\end{aligned}
$$


It is clear that the output window size is directly proportional to the distance of propagation, $z_{0}$, and inversely proportional to the sampling interval $\Delta \xi$ and $\Delta \eta$ in both dimensions. The resultant field recon $\left(m_{x^{\prime}} \Delta \xi^{\prime}, m_{y^{\prime}} \Delta \eta^{\prime}\right)$ is the numerical representation of a complex optical wavefield and from it the intensity image Image $\left(m_{x^{\prime}} \Delta \xi^{\prime}, m_{y^{\prime}} \Delta \eta^{\prime}\right)$ and the phase $\phi\left(m_{x^{\prime}} \Delta \xi^{\prime}, m_{y^{\prime}} \Delta \eta^{\prime}\right)$ can be determined as

$$
\operatorname{Image}\left(m_{x^{\prime}} \Delta \xi^{\prime}, m_{y^{\prime}} \Delta \eta^{\prime}\right)=\left|\operatorname{recon}\left(m_{x^{\prime}} \Delta \xi^{\prime}, m_{y^{\prime}} \Delta \eta^{\prime}\right)\right|^{2},
$$

and

$$
\phi\left(m_{x^{\prime}} \Delta \xi^{\prime}, m_{y^{\prime}} \Delta \eta^{\prime}\right)=\tan ^{-1}\left(\frac{\operatorname{Im}\left\{\operatorname{recon}\left(m_{x^{\prime}} \Delta \xi^{\prime}, m_{y^{\prime}} \Delta \eta^{\prime}\right)\right\}}{\operatorname{Re}\left\{\operatorname{recon}\left(m_{x^{\prime}} \Delta \xi^{\prime}, m_{y^{\prime}} \Delta \eta^{\prime}\right)\right\}}\right)
$$

respectively, where $\operatorname{Im}\{\}$ represents the imaginary part and $\operatorname{Re}\{\}$ the real part of the resultant field recon $\left(m_{x^{\prime}} \Delta \xi^{\prime}, m_{y^{\prime}} \Delta \eta^{\prime}\right)$. Schnars [3] notes that a $\mathrm{DH}$ reconstruction has an advantage over an optical reconstruction in that it has phase information.

The second reconstruction method we tested is the spectral method [34]. This is based on the definition of the Fresnel transform as a chirp multiplication in the Fourier domain, i.e., recon $\left(x^{\prime}, y^{\prime}\right)$ is related to $O(x, y)$ as follows;

$$
\operatorname{recon}\left(x^{\prime}, y^{\prime}\right)=F^{-1}\left\{F\{O(x, y)\} \exp \left[j \pi \lambda z_{0}\left(u^{2}+v^{2}\right)\right]\right\},
$$

where and $u$ and $v$ are spatial frequencies in the $x$ and $y$ directions. The discrete version consists of taking the DFT of the sampled object signal, multiplying it by a sampled version of the quadratic phase version factor and then performing an inverse DFT on the result.

$$
\operatorname{recon}\left(m_{x^{\prime}} \Delta \xi^{\prime}, m_{y^{\prime}} \Delta \eta^{\prime}\right)=D F T^{-1}\left\{D F T\left\{O\left(n_{x} \Delta \xi, n_{y} \Delta \eta\right)\right\} \times \exp [A]\right\}
$$

where $A=j \pi z_{0} \lambda\left(\left(\frac{m_{x}}{N_{x} \Delta \xi}\right)^{2}+\left(\frac{m_{y}}{N_{y} \Delta \eta}\right)^{2}\right)$ and $m_{x}, m_{y}, n_{x}, n_{y}, m_{x^{\prime}}$ and $m_{y^{\prime}}$ all have the same range as previously defined. In the case of the spectral method the output sampling interval $\Delta \xi^{\prime}$ and $\Delta \eta^{\prime}$ are equal to the input sampling intervals, i.e. the pixel size, $\Delta \xi$ and $\Delta \eta$. Using the spectral method for reconstruction, we transform the $\mathrm{DH}$ from the spatial domain into the spatial frequency domain where it is multiplied by the free space transfer 


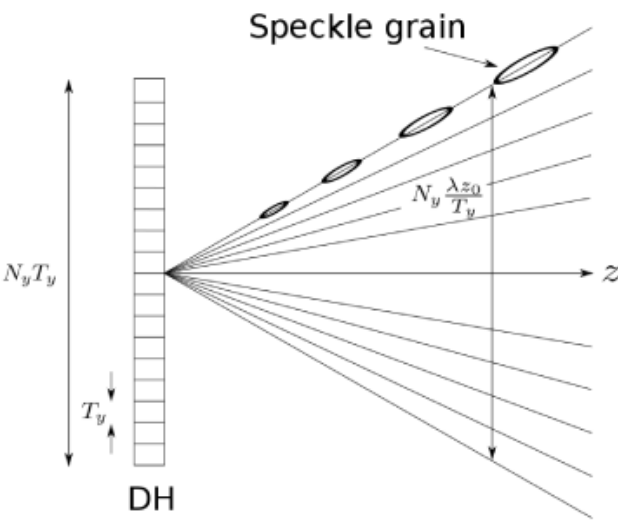

(a)

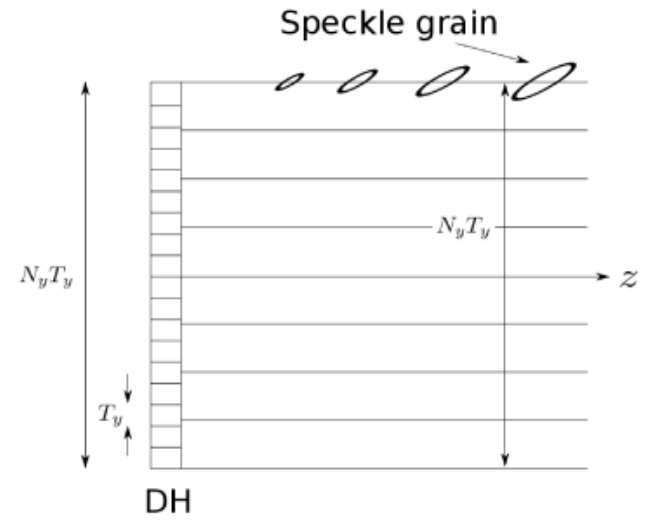

(b)

Figure 1: Output width when reconstructing with the (a) direct method and the (b) spectral method. Also shown is the difference between how both methods average along lines when we sum a series of reconstructions in the $z$ direction. In the case of the direct method averaging occurs along lines that are parallel with the direction of the speckle, whereas in the case of the spectral method the lines cut through the speckle. Adapted from Hennelly et al. [35]

function. We then use the inverse DFT to return to the spatial domain. The result is an image with the same resolution as the CCD, and thus equivalent pixel size. The direct method is often used to reconstruct far field diffraction patterns and the spectral method is usually preferable when reconstructing near field diffraction patterns, with some overlap between the two methods.

We now discuss the differences between the direct and spectral methods of reconstruction in the context of calculating a 3D field. Both methods calculate the Fresnel transform in different ways, allowing us to numerically simulate free space propagation of light in the paraxial approximation. They differ primarily in the output range that they display and the sampling rate at the output plane. For the direct method the output range is proportional to the distance, whereas the spectral method has an output range equal to the size of the CCD camera. However, the manner in which these approaches calculate planes in the $z$ direction is important for the work presented in this paper. Figure 1 (a) shows a visualisation of the direct reconstruction method. The output range is proportional to the reconstruction distance $\lambda z_{0} / \Delta \xi$ in the $x$ direction and $\lambda z_{0} / \Delta \eta$ in the $y$ direction. For the spectral method, shown 
in Fig. 1 (b), the output window is the width of the CCD, $N_{y} \Delta \eta$ [35]. These details are important when it comes to choosing a reconstruction method to use for a given object size. A general rule of thumb is that if the object is larger than the CCD, the direct method should be employed. Otherwise costly zero padding of the $\mathrm{DH}$ is required as a pre-processing step before reconstruction to ensure no aliasing. In both cases $N_{x} N_{y}$ lines emanate from the $\mathrm{DH}$ and cut through the $x, y, z$ coordinate system. In the case of the direct method the propagation is performed radially from each pixel of the DH. In the case of the spectral method the propagation is performed using a cylindrical model of propagation, with each line traveling orthogonal to the $\mathrm{DH}$. These lines therefore propagate through and sample the 3D speckle field differently.

Figure 1 (a) shows that for the direct method the speckle grains are parallel to along the lines emanating from the CCD plane and therefore we expect that the calculated speckle size should closely match that given by the theory [36]. However, for the spectral method [see Fig. 1 (b)] the lines propagate at an angle to the speckle grains and thus we expect that the calculated speckle size may be smaller than that predicted by theory. In the next section we test this hypothesis and discuss how it affects our filtering algorithm.

\subsection{Speckle size and independent speckle patterns}

The addition of independent speckle patterns on an intensity basis will reduce the speckle index by $1 / \sqrt{M}$ where $M$ is the number of independent speckle patterns [4]. The assumption here is that the $M$ images are statistically independent. To satisfy this assumption we shift the reconstruction by an amount at least as large as the average speckle size in a given dimension. Speckle can then be reduced by adding together the resulting intensity reconstructions.

In the $x$ and $y$ directions the theoretical speckle size, given by Good$\operatorname{man}[4]$, is

$$
S_{x, y}=0.9 \lambda\left(\frac{z_{0}}{L_{x, y}}\right),
$$

where $L$ in the length of the sensor in the respective direction and $\lambda$ is wavelength of the capturing light. In the $z$ direction the theoretical speckle size, according to Leushacke and Kirchner [36], is 


$$
S_{z}=\frac{7.31 \sqrt{2} \lambda z_{0}^{2}}{\sqrt{L_{x}^{4}+L_{y}^{4}}} .
$$

As long as $z_{0}$ is large compared with the size of the sensor, then the speckle will have a much greater size in the $z$ direction than in the $x$ or $y$ directions.

In order to determine the physical size of the speckle in a numerical reconstruction of a digital hologram of a planar test object (a DH of a resolution chart) we apply auto-correlation to a uniform area of the reconstruction. In order to validate our result we compare it to the theoretical values. A uniform square feature in the reconstructed image is extracted and auto-correlated [see Box A in Fig. 2 (a)]. The DH was interpolated by a factor of 4 in order to be able to sample at a scale that was significantly smaller than the the expected theoretical speckle size. For the direct reconstruction method interpolation of the reconstruction was achieved by zero padding the $\mathrm{DH}$ prior to reconstruction, whereas for the spectral method interpolation was achieved by zero padding the $\mathrm{DH}$ in the frequency domain using two DFT operations [37]. For the resolution chart hologram [see DH 1 in Table 2 for details of its parameters] the expected speckle size in the $x$ and $y$ directions is $7.16 \mu \mathrm{m}$ and $9.59 \mu \mathrm{m}$, respectively. Application of the direct method to this hologram without interpolation provides a reconstructed image with a sampling interval of $7.96 \mu \mathrm{m}$ and $10.6 \mu \mathrm{m}$ in $x$ and $y$, which is approximately equal to the speckle size in $x$ and $y$. After interpolation, the sampling interval reduces to $1.99 \mu \mathrm{m}$ and $2.66 \mu \mathrm{m}$, which is significantly less than the speckle size. Similarly direct application of the spectral method to the hologram without interpolation provides a reconstructed image with a sampling interval of $6.45 \mu \mathrm{m}$ in $x$ and $y$ (as the pixel sizes have equal extent in $x$ and $y$ ), which once again is close to the size of the speckle in $x$ and $y$. After interpolation the sampling interval reduces to $1.61 \mu \mathrm{m}$ in both directions, which is, again, significantly less than the speckle size. Thus, by interpolating appropriately for both reconstruction methods, we have ensured a sampling interval that is sufficiently less than the expected speckle size and this will allow for an accurate analysis of the speckle in the reconstructed image.

\subsection{Gaussian filtering and our model}

Gaussian filtering is often chosen for image de-noising [38]. The advantage of the Gaussian kernel over the rectangular kernel (mean filtering) is that no 


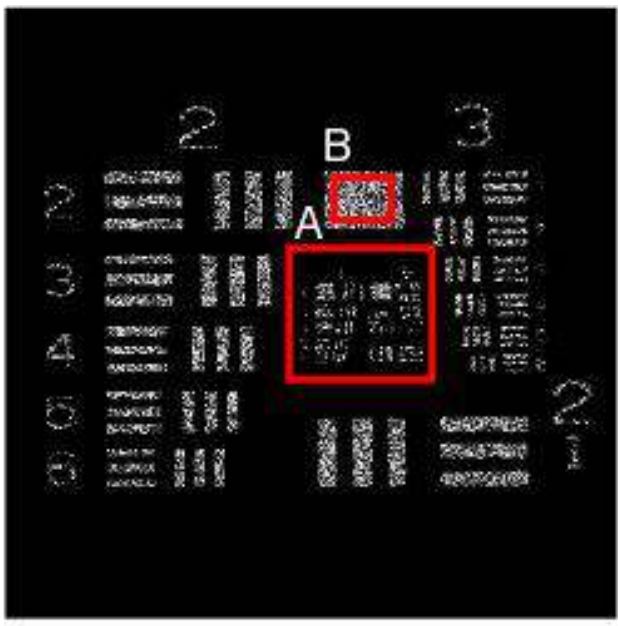

(a)

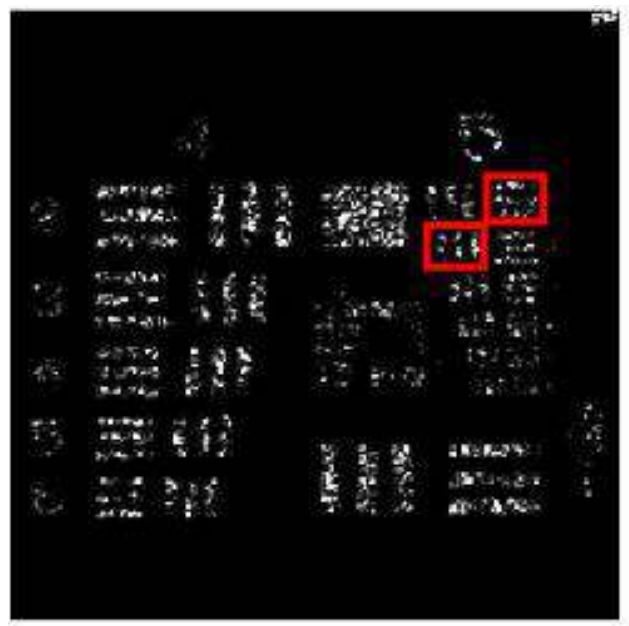

(b)

Figure 2: The USAF resolution chart used to quantify the loss of resolution. (a) The full chart, and (b) a zoomed in section of smaller details (these are marked with region of interest box A in (a)). The chart has a fully developed speckle pattern (speckle index is calculated on the homogeneous section marked with region of interest box B in (a)) and using visual inspection the minimum resolvable bars in $x$ and $y$ is highlighted by the two dashed white lined rectangles in (b).

nulls occur in the frequency domain. This can be explained as follows; the FT of a Rect function is a Sinc function which contains a series of null (zero value) points in its two dimensional distribution. Since convolution in space with a Rect function is equivalent to multiplication in frequency by a Sinc function we can expect the FT of the filtered image to contain a number of null frequencies which will inevitably distort the image. Alternatively the FT of a Gaussian function is also a Gaussian function which reduces smoothly in value, but does not contain null points. A 3D Gaussian distribution has the following form

$$
G(x, y, z)=A \exp \left[-\left(\frac{x^{2}}{2 \sigma_{x}^{2}}+\frac{y^{2}}{2 \sigma_{y}^{2}}+\frac{\left(z-z_{0}\right)^{2}}{2 \sigma_{z}^{2}}\right)\right]
$$

where $\sigma_{x}, \sigma_{y}$ and $\sigma_{z}$ are the standard deviations in the (x,y,z) directions, $A$ is a normalization factor $1 /\left(\sqrt{2 \pi \sigma_{x}} \sqrt{2 \pi \sigma_{y}} \sqrt{2 \pi \sigma_{z}}\right)$ and $z_{0}$ is the reconstruction distance. There exists no obvious direct relationship between the size of the Gaussian kernel and the number of independent speckles that will be averaged together resulting from the convolution. In order to simplify our analysis we 
attempt to apply an approximately direct relationship between the standard deviation of the Gaussian function and the reduction in speckle index. To do this we empirically investigate 2D Gaussian filtering of a test hologram of a USAF resolution chart with a range of different standard deviation values. Our assumption is that our model will be similar to the model used for predicting the speckle index when multiple independent speckle patterns are added together. In that case the speckle index is given by $1 / \sqrt{M}$ where $M$ statistically independent images are added together [4]. In this case we assume that Gaussian filtering will result in a speckle index given by $1 / \sqrt{M_{x}}$, where $M_{x}$ is a linear function of $\sigma_{x}$. In order to derive this function we use two expected values. The first is when $\sigma_{x} \rightarrow 0$, the speckle index, $C$, should be 1 indicating no speckle reduction. Thus as the Gaussian function narrows to approximate a Dirac delta function, it allows the input to pass through unimpeded and there will only be one independent speckle pattern giving $C=1$. Upon investigation of our experimental measurements a second point of interest was chosen, this time with $\sigma_{x}=S_{x}$ and $C \approx \frac{1}{2}$. Therefore we conclude that an approximate model for the speckle index resulting from 1D Gaussian filtering in the $x$ direction is given by

$$
C=\frac{1}{\sqrt{1+\frac{3 \sigma_{x}}{S_{x}}}} .
$$

A similar model can be used for 1D filtering in both the $y$ and $z$ directions. Extension to the $2 \mathrm{D}$ case is given by the product of the $1 \mathrm{D}$ values,

$$
C=\frac{1}{\sqrt{1+\frac{3 \sigma_{x}}{S_{x}}}} \times \frac{1}{\sqrt{1+\frac{3 \sigma_{y}}{S_{y}}}}
$$

and it follows that the 3D case is given by,

$$
C=\frac{1}{\sqrt{1+\frac{3 \sigma_{x}}{S_{x}}}} \times \frac{1}{\sqrt{1+\frac{3 \sigma_{y}}{S_{y}}}} \times \frac{1}{\sqrt{1+\frac{3 \sigma_{z}}{S_{z}}}} .
$$

The theoretical values for the speckle index resulting from 1D, 2D and 3D Gaussian filtering will be presented in Sect. 4 and as will be readily seen they closely agree with the experimental results. 


\section{Metrics and theoretical resolution}

We use speckle index, also known as speckle contrast, together with a resolution metric to investigate the results. Fig. 2 shows a reconstruction of a $\mathrm{DH}$ (with no speckle reduction applied) of a USAF resolution chart, purchased from Edmund Optics, which is opaque except for the transparent areas [39]. Deciding upon the resolution is a subjective process involving visual inspection. In order to ensure a consistent measurement of resolution across the entire data set we define a set of measurement criteria for the detection of a minimum value. The minimum resolvable set of lines must obey the following rules;

1. There should be clear separation between the bars in a given group of three, i.e., the contrast between the bars and the space between the bars should be strong enough to preclude any doubt that there is a gap between the bars.

2. Individual bars do not need to be contiguous and can be corrupted by speckle noise.

3. Each set of bars with a lower resolution than the chosen set should also be resolvable.

4. A set of bars is not penalized if a neighboring set of bars blurs into it.

5. If it is not clear what the resolution is between two sets of bars then the lower resolution is chosen.

When no speckle reduction is applied the resolution, seen in Fig. 2 (b), in the $x$ direction (vertical bars) is group 5 element 1, or 35.91 line pairs per millimeter, and the resolution in the $y$ direction (horizontal bars) is group 5 element 2, or 32 line pairs per millimeter. The resolution, $R$, which denotes the of the number of lines per millimeter is given by $R=2^{G+(E-1) / 6}$, where $\mathrm{G}$ is the group and $\mathrm{E}$ the element within that group. Groups 2 and 3 both containing elements 1 through 6 are clearly visible in Fig. 2 (a). For the remainder of the paper the resolution is given in line pairs per millimeter.

We now discuss the expected theoretical resolution for the resolution $\mathrm{DH}$. The approximate bandwidth limit for a DH system is imposed by the aperture of the camera, $W_{C C D x, y}$, the wavelength of the laser used in the system, $\lambda$, and the object camera distance, $z_{0}$ [39];

$$
\text { Bandwidth }_{x, y} \cong \frac{W_{C C D x, y}}{\lambda z_{0}}
$$


where the $x, y$ subscript denotes that the equation is valid for both the $x$ and the $y$ parameters. We can rewrite the equation above in terms of system parameters

$$
\begin{aligned}
\text { Bandwidth }_{x} \cong \frac{N_{x} \Delta \xi}{\lambda z_{0}}, \\
\text { Bandwidth }_{y} \cong \frac{N_{y} \Delta \eta}{\lambda z_{0}} .
\end{aligned}
$$

Therefore we can expect a complex image that is recorded by a DH system will have a frequency range in $f_{x}$ and $f_{y}$ (spatial frequency coordinates corresponding to $x$ and $y$ respectively) given by;

$$
\begin{aligned}
& \left|f_{x}\right| \lesssim \frac{N_{x} \Delta \xi}{2 \lambda z_{0}}, \\
& \left|f_{y}\right| \lesssim \frac{N_{y} \Delta \eta}{2 \lambda z_{0}} .
\end{aligned}
$$

The maximum recoverable spatial frequency is given by

$$
\begin{aligned}
& f_{x_{\text {max }}} \cong \frac{N_{x} \Delta \xi}{2 \lambda z_{0}}, \\
& f_{y_{\text {max }}} \cong \frac{N_{y} \Delta \eta}{2 \lambda z_{0}} .
\end{aligned}
$$

We note that the maximum recordable frequency is directly proportional to the camera width in the direction of interest and is inversely proportional to the wavelength and the camera object distance. We also note that there are at least three other factors that further reduce the bandwidth of the system [39]; these include (i) the averaging effect of the pixel, which for a Fresnel system amounts to an averaging of the complex reconstruction; (ii) the effect of sampling which can result in overlapping replicas of the reconstruction; and (iii) the pixel quantization, which can lead to errors in the reconstructed image [40]. Furthermore, we expect the speckle noise (and indeed other sources of noise) to further impact on resolution. Thus, it should be stated that the limits given above in Eqs. 24 and 25 are approximate and should be treated as upper limits. In practice we can expect that the maximum recordable frequencies will be less than the values determined by Eqs. 24 and 25. 
The unit of lines per millimeter can be related to maximum recoverable frequency of the system. This unit is based on the number of bright and dark line pairs that can be found in a length of $1 \mathrm{~mm}$. Equations 24 and 25 can be interpreted as the number of bright and dark lines that can be found in a length of $1 \mathrm{~m}$, assuming that all of the parameters in the equations are defined in meters. Therefore we can redefine the theoretical maximum frequencies in terms of the pairs per millimeter as follows

$$
\begin{aligned}
& f_{x_{\text {max }}} \cong \frac{N_{x} \Delta \xi}{2 \lambda z_{0}}, \\
& f_{y_{\text {max }}} \cong \frac{N_{y} \Delta \eta}{2 \lambda z_{0}} .
\end{aligned}
$$

For the resolution chart $\mathrm{DH}$, the parameters of which are defined in Table 2 (DH 1), and we can calculate the values of the theoretical maximum frequencies to be

$$
\begin{aligned}
& f_{x_{\text {max }}} \cong 62.85 \text { (line pairs / mm), } \\
& f_{y_{\text {max }}} \cong 46.95 \text { (line pairs / mm). }
\end{aligned}
$$

As expected the experimental values of 35.91 and 32 lines pairs / mm respectively fall some way short of the expected theoretical maximum values. We add one final note. If we are correct in our previous assumption that the speckle noise will impact on the maximum recordable frequencies, then we can expect that speckle reduction may increase the number of line pairs per millimeter that can be observed in the image.

\section{Experimental results}

In order to test our approach, reconstructions of the resolution chart $\mathrm{DH}$ introduced in the previous section were used to quantify the improvement in speckle contrast and the resulting loss in resolution. We investigated $1 \mathrm{D}$ filtering in the $x, y$ and $z$ directions separately, $2 \mathrm{D}$ filtering in the $x y$ plane, and finally full 3D filtering in all three spatial directions. In all cases resolution results are provided separately for $x$ and $y$. We provide evidence that our model closely approximates the theory in terms of speckle reduction

for a given number of independent speckle patterns. We also provide evidence 


\begin{tabular}{|c|c|c|c|c|c|c|}
\hline $\mathrm{DH}$ & $\lambda(\mu \mathrm{m})$ & $\Delta \xi(\mu \mathrm{m})$ & $\Delta \eta(\mu \mathrm{m})$ & $N_{x}$ & $N_{y}$ & $z_{0}(\mathrm{~m})$ \\
\hline 1 & 0.785 & 6.45 & 6.45 & 1392 & 1040 & 0.091 \\
\hline 2 & 0.785 & 6.45 & 6.45 & 1392 & 1040 & 0.279 \\
\hline 3 & 0.785 & 6.45 & 6.45 & 1392 & 1040 & 0.3 \\
\hline 4 & 0.785 & 6.45 & 6.45 & 1392 & 1040 & 0.394 \\
\hline 5 & 0.785 & 3.45 & 3.45 & 2448 & 2050 & 0.105 \\
\hline
\end{tabular}

Table 2: Parameter details for DHs used in the experiments. The numbers 1 through 5 are labels for DHs of the USAF resolution chart, two screws, a toy spindle, a stormtrooper object and two small chairs. $\lambda$ : wavelength of the light used, $\Delta \xi$ : sensor pixel size in $x$, $\Delta \eta$ : sensor pixel size in $y, N_{x}, N_{y}$ : number of pixels on the sensor in $x$ and $y$ respectively, $z_{0}$ : distance of the object to the sensor.

\begin{tabular}{|c|c|c|c|}
\hline DH & $S_{x}(\mu \mathrm{m})$ & $S_{y}(\mu \mathrm{m})$ & $S_{z}(\mu \mathrm{m})$ \\
\hline 1 & 7.16 & 9.59 & 729 \\
\hline 2 & 21.2 & 29.4 & 6800 \\
\hline 3 & 23.6 & 31.6 & 7900 \\
\hline 4 & 31 & 41.5 & 13600 \\
\hline 5 & 8.74 & 10.43 & 1000 \\
\hline
\end{tabular}

Table 3: Theoretical speckle size for each DH. $S_{x}, S_{y}$ and $S_{z}$ are the sizes in $x, y$ and $z$ respectively. These values are calculated using Eqs 13 and 14, and the DH parameters given in Table 2.

that an improvement in the depth of focus can be achieved when filtering in the $z$ direction, if the object of interest has an appreciable extent in this direction.

Following our analysis with the resolution chart DH, we investigate the performance of the method on a number of different holograms. Tables 2 and 3 provide details such as sensor pixel sizes, number of pixels on the sensor, reconstruction distances, wavelength of the light used during capture and the theoretical speckle sizes (based on Eqs. 13 and 14) for the DHs tested in this paper. Table 4 gives details of the sampling intervals in and around the reconstruction plane used in all three directions for both reconstruction methods (direct and spectral method). We note here again that the sampling interval in the $z$ direction is calculated using the concept of depth of focus given by Kreis [5]. In Sect. 2.3 we postulated that the effective speckle size could be smaller in the $z$ direction when the images are stacked using the spectral method because the direction of the lines along which the averaging 


\begin{tabular}{|c|c|c|c|c|c|c|}
\hline & \multicolumn{3}{|c|}{ Direct Method } & \multicolumn{3}{c|}{ Spectral Method } \\
\hline DH & $T_{x}(\mu \mathrm{m})$ & $T_{y}(\mu \mathrm{m})$ & $T_{z}(\mu \mathrm{m})$ & $T_{x}(\mu \mathrm{m})$ & $T_{y}(\mu \mathrm{m})$ & $T_{z}(\mu \mathrm{m})$ \\
\hline 1 & 7.96 & 10.66 & 161.64 & 6.45 & 6.45 & 175.19 \\
\hline 2 & 24.39 & 32.65 & 1500 & 6.45 & 6.45 & 536.54 \\
\hline 3 & 26.23 & 35.1 & 1800 & 6.45 & 6.45 & 576.92 \\
\hline 4 & 34.45 & 46.11 & 3000 & 6.45 & 6.45 & 757.69 \\
\hline 5 & 9.71 & 11.59 & 240.37 & 3.45 & 3.45 & 101.95 \\
\hline
\end{tabular}

Table 4: Sampling intervals in $x, y$ and $z$ for both the direct and spectral methods for the DHs used in this paper. $T_{x}$ : Sampling interval in $x, T_{y}$ : Sampling interval in $y$, $T_{z}$ : Sampling interval in $z$ (given by the depth of focus). We note that these are the calculated theoretical sampling intervals and that these are reduced further to ensure sufficient sampling of the $3 \mathrm{D}$ field.

occurs in the $z$ direction is different to the direction of the speckle and indeed in Sect. 2.4 we measured a slightly smaller speckle size. For the experiments carried out on the resolution chart DH we do not adapt the size of the Gaussian kernels to take this into account as the difference in speckle size was quite small due to the small camera object distance $(0.091 \mathrm{~m})$ at which the resolution chart was recorded. For larger camera object distances this effect is more pronounced and the size of the filter used should be adapted accordingly if the spectral method is used to reconstruct the DH. As all of our tests were carried out on the resolution chart DH, we determined the width of the Gaussian kernel based on the dimensions of the theoretical speckle size (given in Table 3, DH 1). Table 4, by giving values for the depth of focus for all DHs used, also gives an insight into the size of the sampling period need to ensure sufficient sampling is performed. The values in Table 4 were calculated using the equations given in Table 1 and the parameters in Table 2. In the following sections tests are carried out with the resolution chart $\mathrm{DH}$ and using both the direct and the spectral method of reconstruction.

\section{1. $1 D$ filtering}

Our initial tests involve filtering with Gaussian kernels that have extent in only one direction $(x, y$ or $z$ ). Figure 3 (a) plots the resolution in $x$ and $y$ for $1 \mathrm{D}$ filtering in the $x$ direction. Initially the resolution in $x$ is better than that in $y$ due to the fact that the CCD is rectangular and is larger in the $x$ direction $(0.009 \mathrm{~m}$ versus $0.0067 \mathrm{~m})$ [39]. We observe an improvement in the $y$ resolution, before a slight dip, and then the resolution remains 


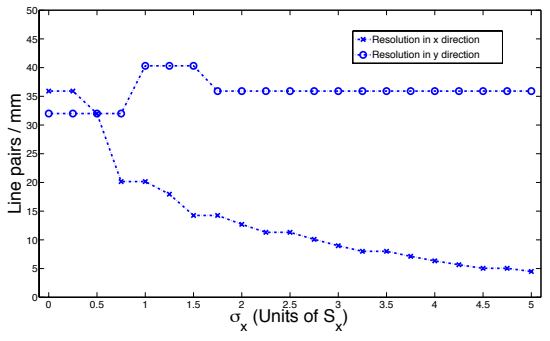

(a)

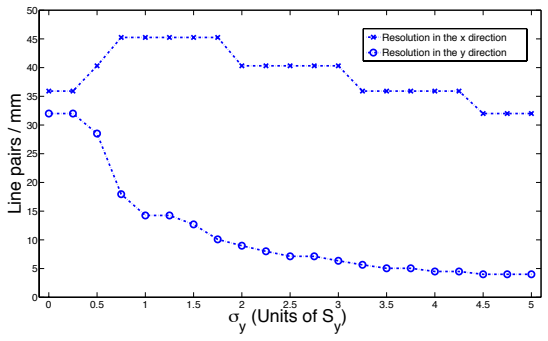

(b)

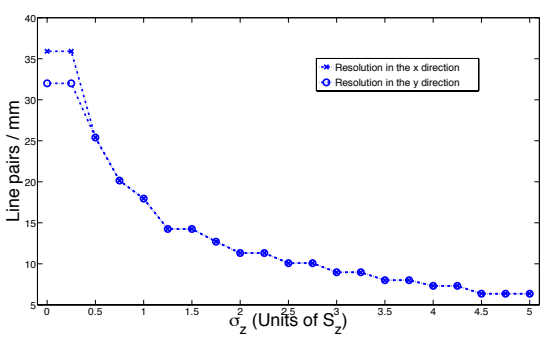

(c)

Figure 3: Resolution in $x$ and $y$ when filtering only in the $x$ direction (a), only in the $y$ direction (b) and only in the $z$ direction (c). These results are applicable to both the direct and spectral methods of reconstruction as the resolution was the same regardless of which method was chosen.

constant at 35.91 line pairs per $\mathrm{mm}$. It is an interesting phenomenon that the resolution can increase through filtering (as was predicted in Sect. 3), but it can be attributed to an improvement in image quality brought about by the reduction in speckle index. As expected, the resolution in $x$ steadily degrades as the width of the Gaussian filter increases until, when $\sigma_{x}=5$ (units of $S_{x}$ ), only 4.49 line pairs per $\mathrm{mm}$ can be observed. Figure 4 shows the effect on speckle index when filtering in the $x, y$ and $z$ directions using both the direct and the spectral methods of reconstruction. It confirms that our model reflects the actual speckle index very well. We note that there are small differences between the direct and spectral methods with the latter method having a slightly smaller speckle index. Figure 5 shows a section of the resulting resolution chart after filtering is applied only in the $x$ direction. Figure 3 (b) plots the resolution in $x$ and $y$ for 1D filtering in the $y$ direction. This time we observe an improvement in resolution in the $x$ direction from 35.91 line pairs per mm up to 45.25 line pairs per $\mathrm{mm}$. However this degrades to 32 lines as the filter size reaches larger sizes (for $\sigma_{y}=4.5 S_{y}$ to $5 S_{y}$ ). The 


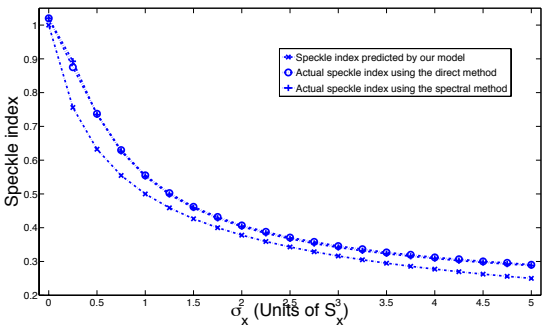

(a)

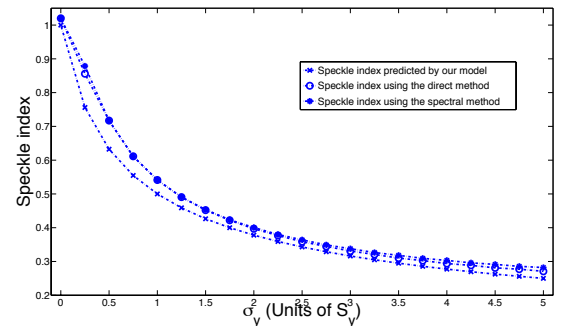

(b)

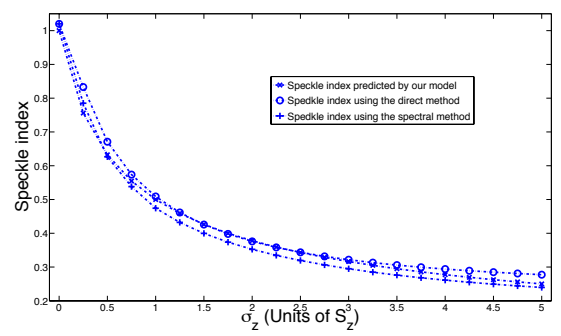

(c)

Figure 4: Speckle index when filtering only in the $x$ direction (a), only in the $y$ direction (b) and only in the $z$ direction (c).

reason for this is that sets of 3 vertical bars start to blur into each other, thus reducing the resolution. Figure 3 (c) plots the resolution in $x$ and $y$ for $1 \mathrm{D}$ filtering in the $z$ direction. As we are averaging along a direction that is orthogonal to both $x$ and $y$ there is no improvement in resolution as the filter kernel size increases, only a degradation. We note that for this method of filtering there was more noise between pairs of bars that we consider resolved than for the similar 1D $x$ and $y$ filtering. We also note that for larger filter kernels this method outperforms the previous two conditions in at least one direction and if the desired goal is to have good resolution in both $x$ and $y$ directions, this could be the method of choice in the event only $1 \mathrm{D}$ filtering is to be carried out. If improved resolution is required is another direction then the Gaussian kernel can be rotated as required.

\section{2. $2 D$ filtering}

In this section, we take the next logical step and filter using a Gaussian kernel with extent in both the $x$ and $y$ direction. Figure 6 (a) plots the resolution in $x$ and $y$ for $2 \mathrm{D}$ filtering in the $x$ and $y$ directions. The resolution 


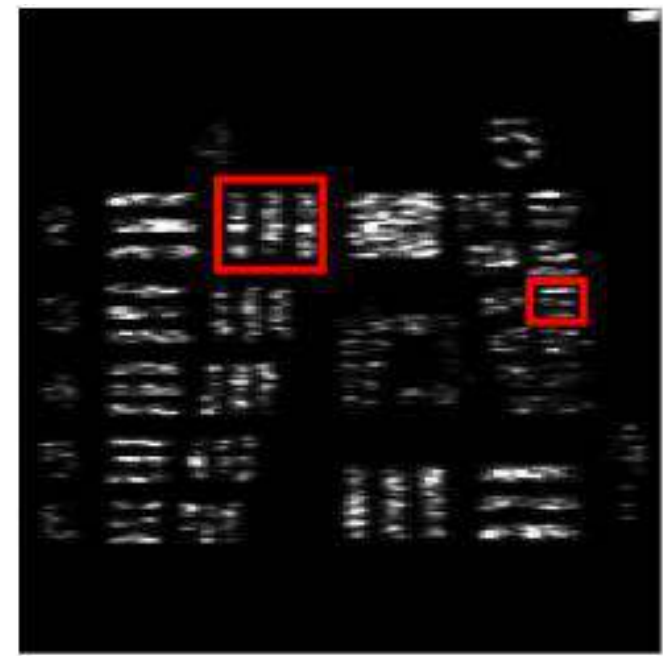

(a)

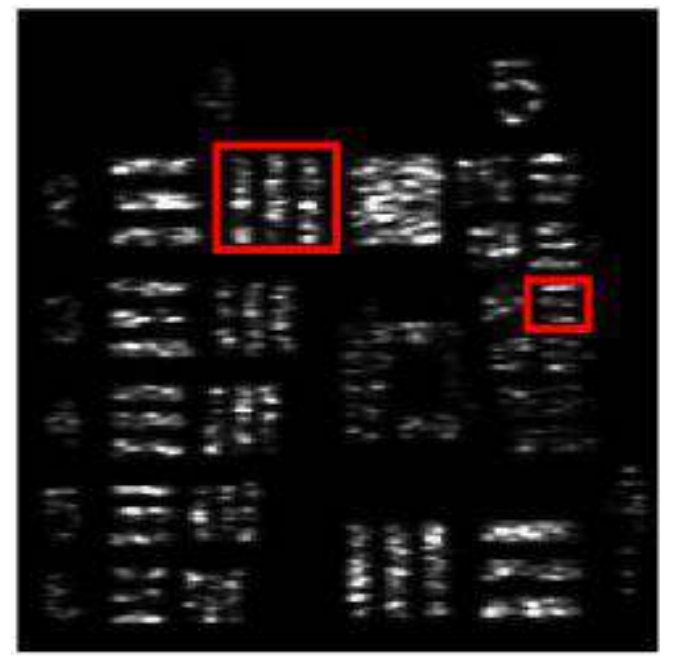

(b)

Figure 5: Filtering only in the $x$ direction with $\sigma_{x}=1.25 S_{x}$ (a) Using the direct method of reconstruction, and (b) using the spectral method of reconstruction. In both reconstruction cases the $x$ resolution is 17.95 line pairs per $\mathrm{mm}$ and the $y$ resolution is 40.31 line pairs per mm (marked by the red boxes). The speckle contrast was reduced to a speckle index of 0.5025 in the case of the direct method and 0.4984 for the spectral method.

in $x$ is better than that in $y$ due to the larger extent of the sensor in the $x$ direction for all tests conducted. We note an initial improvement in the $x$ direction resolution from 35.91 line pairs per $\mathrm{mm}$ (with no filtering) to 40.31 line pairs per $\mathrm{mm}$, and thereafter as the filter kernel increases in size the resolution reduces. At first the resolution in the $y$ direction remains the same as when no filtering is applied at 35.91 line pairs per mm, but then resolution degrades at approximately the same rate as that in $x$. If maintaining resolution in both $x$ and $y$ directions is important, then $2 \mathrm{D}$ filtering provides large gains over 1D filtering. Figure 6 (b) plots the actual speckle index achieved when filtering in 2D against the expected theoretical speckle index. The model once again approximates the theoretical expected speckle index values well. Figure 7 (a) and (b) shows that with $\sigma_{x}$ and $\sigma_{y}$ set to $\frac{S_{x}}{2}$ and $\frac{S_{y}}{2}$, respectively, the $x$ resolution is 40.31 line pairs per $\mathrm{mm}$ and the $y$ resolution is 32 line pairs per $\mathrm{mm}$, and the speckle index is approximately 0.52 . In comparison, if we were to filter in 1D in any direction and our aim was to achieve a similar speckle index (i.e., a value close to $0.5)$, the best case would be filtering in the $x$ direction which provides 20.15 


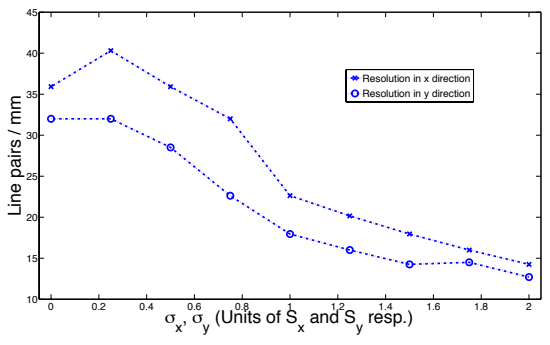

(a)

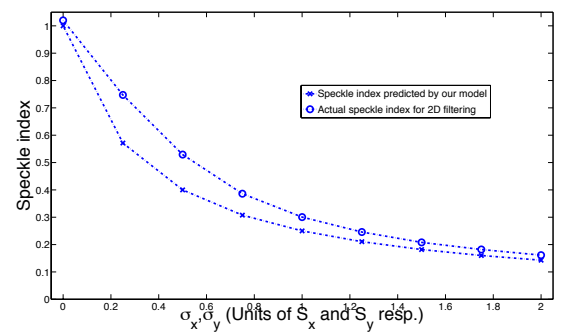

(b)

Figure 6: (a) Resolution in $x$ and $y$ when filtering in 2D. These results are applicable to both the direct and spectral methods of reconstruction as the resolution was the same regardless of which method was chosen. (b) Graph showing the expected theoretical value of the speckle index versus the actual speckle index when filtering in 2D in $x$ and $y$. As there were only negligible differences in speckle index between the direct and spectral reconstruction methods, only the direct method is plotted here

line pairs per $\mathrm{mm}$ in $x$ resolution, while of course having better resolution in the $y$ direction at 40.31 line pairs per mm. Comparing 2D filtering with $1 \mathrm{D}$ filtering is difficult, but a general rule of thumb is that if resolution is required in both spatial directions then $2 \mathrm{D}$ filtering should be chosen over 1D filtering. Furthermore, due to the separability of the Gaussian kernel, the 2D filter can be implemented as a pair of orthogonal 1D Gaussians, which reduces the computational complexity from $O\left(n^{2}\right)$ to $O(2 n)$.

\section{3. $3 D$ filtering}

In this section, we take advantage of the inherent 3D nature of DHs and and filter using a Gaussian kernel with support in the $x, y$ and $z$ directions. Figure 8 (a) plots the resolution in $x$ and $y$ for 3D filtering, where $\sigma_{x}, \sigma_{y}$ and $\sigma_{z}$ are increased by equal multiples of $S_{x}, S_{y}$ and $S_{z}$, respectively. The resolution in $x$ is better than that in $y$ for the first two kernel sizes, but after that the resolution is the same in both directions. The trend is for resolution to degrade in both directions as the kernel size increases in $x, y$ and $z$. Fig 8 (b) plots the actual speckle index achieved when filtering in 3D against the expected theoretical speckle index. We note that there are larger differences between the model and theoretical expected speckle index values than for the $1 \mathrm{D}$ or $2 \mathrm{D}$ filtering cases. Fig. 9 shows a result for $3 \mathrm{D}$ filtering when $\sigma_{x}$, $\sigma_{y}$ and $\sigma_{z}$ are set to $\frac{S_{x}}{2}, \frac{S_{y}}{2}$ and $\frac{S_{z}}{2}$, respectively. If resolution in both the $x$ and $y$ directions is important then $3 \mathrm{D}$ filtering outperforms $1 \mathrm{D}$ filtering. For this example the speckle index is approximately 0.4 for both reconstruction 


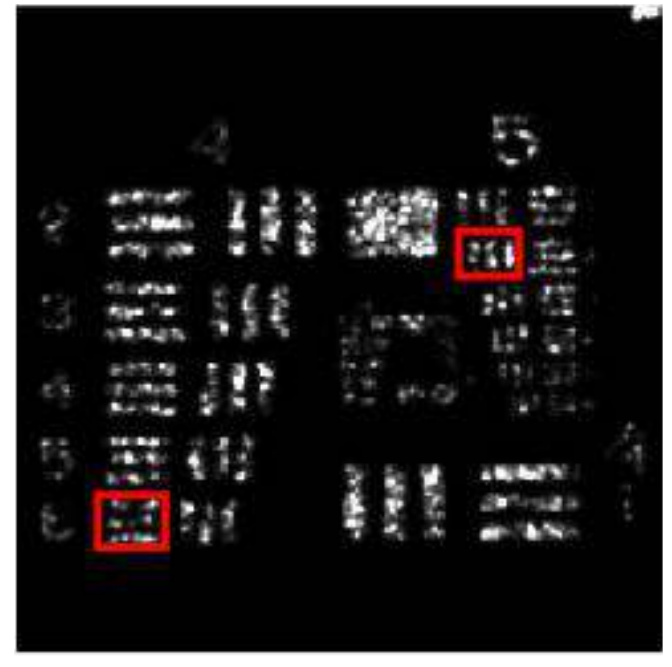

(a)

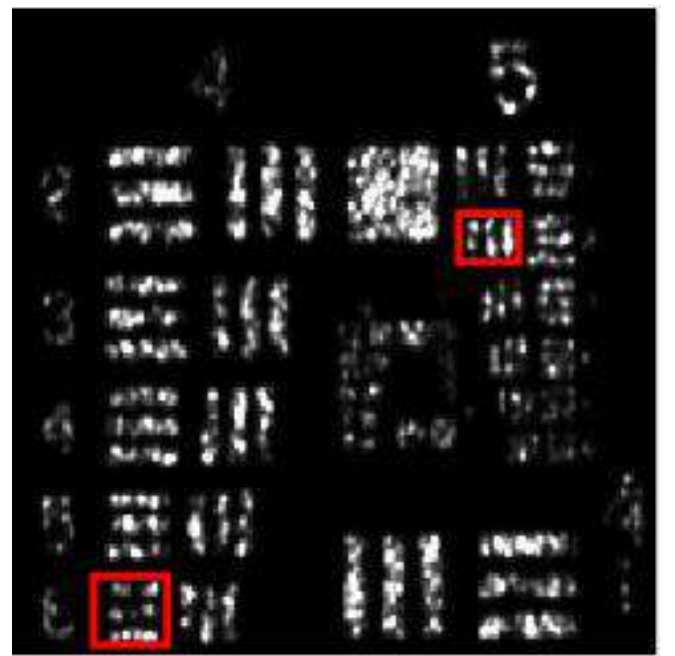

(b)

Figure 7: Filtering in 2D in the $x$ and $y$ directions with $\sigma_{x}=\frac{S_{x}}{2}$ and $\sigma_{y}=\frac{S_{y}}{2}$. (a) Using the direct method of reconstruction (speckle index $=0.5292$ ), and (b) using the spectral method of reconstruction (speckle index $=0.5285$ ). In both cases the $x$ resolution is 35.92 line pairs per $\mathrm{mm}$ and the $y$ resolution is 28.51 line pairs per $\mathrm{mm}$ (marked by the red boxes).

methods and the resolution in $x$ is 25.4 line pairs per $\mathrm{mm}$ and resolution in $y$ is 22.63 line pairs per mm. In comparison, if we were to filter in $1 \mathrm{D}$ in any direction and our aim was to achieve a similar speckle index (i.e., a value close to 0.4 ), the best case would be filtering in the $z$ direction which provides 14.25 line pairs per $\mathrm{mm}$ in both the $x$ and $y$ directions. However, when compared with 2D filtering, 3D filtering performed slightly worse for every test, when the criteria is to have good resolution in both the $x$ and $y$ directions coupled with a low speckle index. We note that the USAF resolution DH is a planar object and thus has no extent in the $z$ direction. We expect $2 \mathrm{D}$ filtering to perform extremely well on such an object. This does not, however, mean that 3D filtering should be dismissed, as it can perform well if the object has extent in the $z$ direction. Then we actually observe, with astute positioning of the filter, an improvement in the depth of focus too.

We also performed 2D and 3D filtering on in-line DHs 2-4 from Tab 2. Figure 10 shows the results when filtering was applied to a stormtrooper DH, a DH of two bolts and a spindle object for the 2D and 3D cases for with different sized Gaussian kernels. In the figure the original reconstruction 


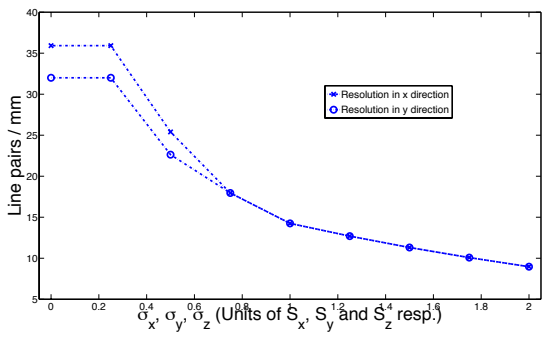

(a)

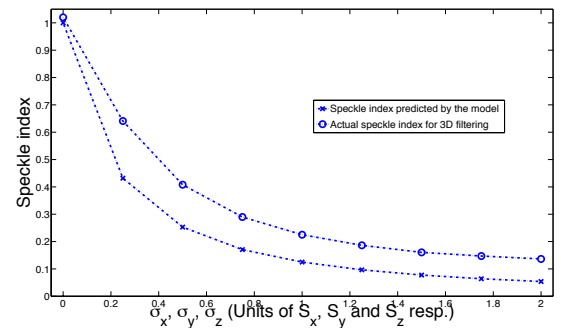

(b)

Figure 8: (a) Resolution in $x$ and $y$ when filtering in 3D. These results are applicable to both the direct and spectral methods of reconstruction as the resolution was the same regardless of which method was chosen. (b) Graph showing the expected theoretical value of the speckle index versus the actual speckle index when filtering in 3D in the $x, y$ and $z$ directions.

is shown with no speckle reduction applied, then the result of applying 2D filtering with $\sigma_{x}=2 S_{x}$ and $\sigma_{y}=2 S_{y}$ and finally the result of applying 3D filtering with $\sigma_{x}=1.75 S_{x}, \sigma_{y}=1.75 S_{y}$ and $\sigma_{z}=1.75 S_{z}$. The resulting speckle index for the reconstructions with $2 \mathrm{D}$ filtering applied is $\approx 0.14$ (theoretical value given by our model is 0.16 ) and for the reconstructions with $3 \mathrm{D}$ filtering applied is $\approx 0.09$ (theoretical value is 0.064 ). Overall the 3D filtering results [see Fig. 10 (c), (f) and (i)] are slightly more blurred, but have a lower speckle index, than the 2D filtering results [see Fig. 10 (b), (e) and $(\mathrm{h})]$

Although our quantitative results using the resolution chart DH revealed no benefit in choosing 3D filtering over $2 \mathrm{D}$ filtering, the ability to average in another dimension can be advantageous under certain circumstances. Filtering in the $z$ direction with objects that have extent in that direction can improve the depth of focus of resulting image. To illustrate this we investigated filtering a DH of two small chairs in the $z$ direction and provide qualitative evidence that the depth of focus is indeed improved. The DH was recorded with the parameters shown in row 5 in Table 2. The same laser was used to record this hologram as in the case of the resolution chart but the recording distance was a little further from the camera at $0.105 \mathrm{~m}$. We note that the expected speckle size is a little larger than for the resolution chart as shown in Table 3. The speckle size in the $x$ and $y$ directions are given by $8.74 \mu \mathrm{m}$ and $10.43 \mu \mathrm{m}$ respectively, while $S_{z}$ is equal to $1 \mathrm{~mm}$. This particular object has a large depth; The front of the object is in focus at a 


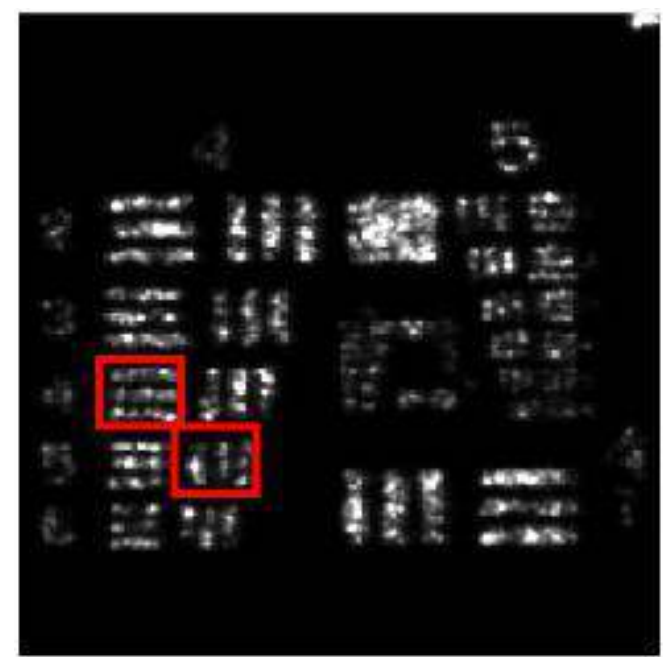

(a)

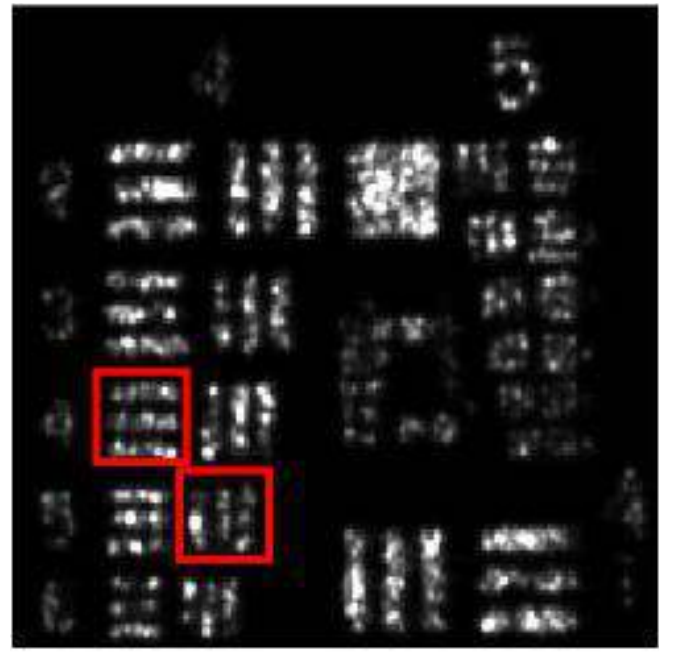

(b)

Figure 9: Filtering in 3D in the $x, y$ and $z$ directions with $\sigma_{x}=\frac{S_{x}}{2}, \sigma_{y}=\frac{S_{y}}{2}$ and $\sigma_{z}=\frac{S_{z}}{2}$. (a) Using the direct method of reconstruction (speckle index $=0.4079$ ), and (b) using the spectral method of reconstruction (speckle index $=0.4037$ ). In both reconstruction cases the $x$ resolution is 25.4 line pairs per $\mathrm{mm}$ and the $y$ resolution is 23.63 line pairs per $\mathrm{mm}$ (marked by the red boxes).

reconstruction distance of $0.0975 \mathrm{~m}$ as shown in Fig. 11 (a), while the back of the object is in focus at a reconstruction distance of $0.1125 \mathrm{~m}$ as shown in Fig. 11 (b), which gives us an object depth of $1.5 \mathrm{~cm}$ and a mid-point reconstruction distance of $z_{0}=0.105 \mathrm{~m}$. Both of these reconstructions were calculated after first zero padding the DH up to a size of $6150 \times 7344$ pixels and reconstructing using the direct method. The resulting image in Fig. 11 (a) has a pixel size of $3.61 \mu \mathrm{m}$ and $3.02 \mu \mathrm{m}$ in the $x$ and $y$ dimensions respectively, and in Fig. 11 (b) has a pixel size of $4.16 \mu \mathrm{m}$ and $3.48 \mu \mathrm{m}$ in the $x$ and $y$ dimensions respectively. In both Fig. 11 (a) and (b) we show zoomed in regions of the reconstruction which highlight small features in the front and back parts of the object. We note that no filtering was applied to either of these reconstructions.

It is interesting to apply our 3D filtering algorithm with a Gaussian filter that has a standard deviation that is wide enough to cover a large range of the full object depth. We implement the method with $\sigma_{x}=S_{x}, \sigma_{y}=S_{y}$ and $\sigma_{z}=7 S_{z}$. The result of using this 3D filter using the direct method is shown in Fig. 12 (a), where we have chosen the reconstruction distance to be 


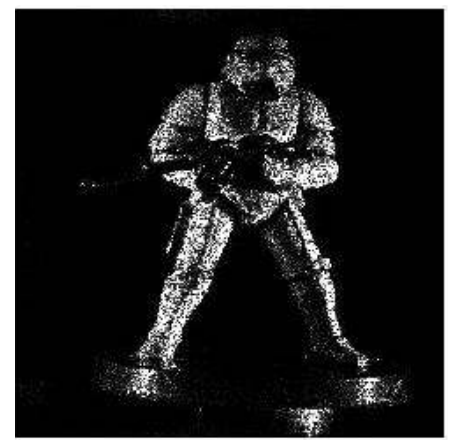

(a)

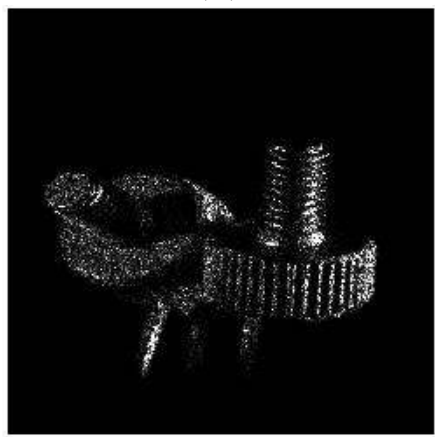

(d)

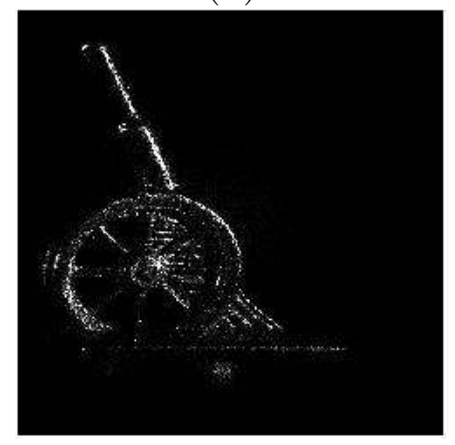

(g)

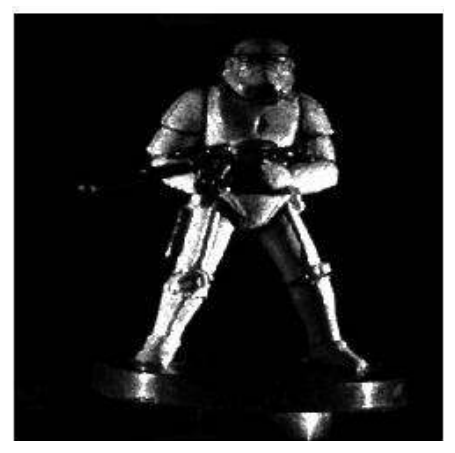

(b)

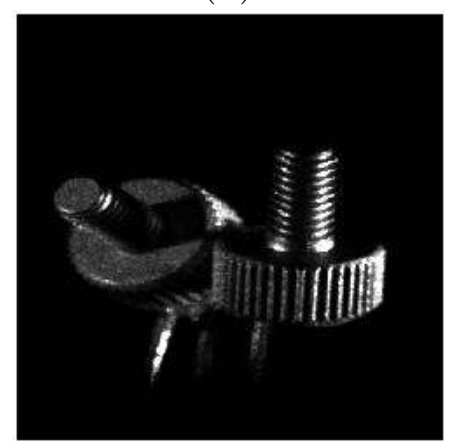

(e)

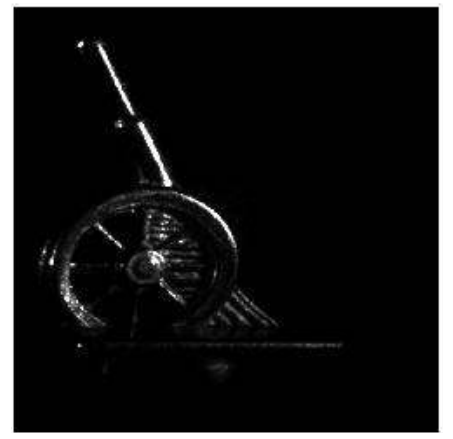

(h)

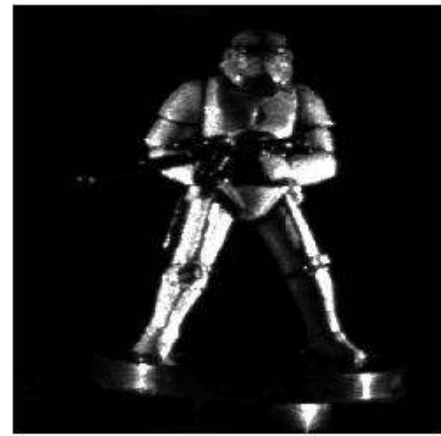

(c)

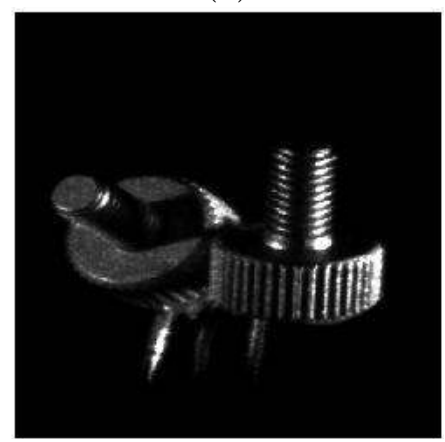

(f)

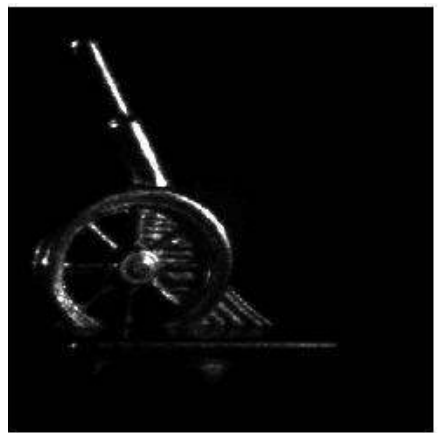

(i)

Figure 10: Filtering in 2D and 3D applied to three different DHs (an in-line DH of a stormtrooper object, two bolts and a spindle object). (a), (d) and (g) show the the original reconstructions with no speckle reduction applied (all have a speckle index of $\approx 1.0$ ). (b), (e) and (h) shows 2D filtering applied with parameters $\sigma_{x}=2 S_{x}$ and $\sigma_{y}=2 S_{y}$ (speckle index for these figures is $\approx 0.14$ ) and finally (c), (f) and (i) shows 3D filtering applied with parameters $\sigma_{x}=1.75 S_{x}, \sigma_{y}=1.75 S_{y}$ and $\sigma_{z}=1.75 S_{z}$ (speckle index for these figures is $\approx 0.09$ ). 
the midpoint object distance $z_{0}=0.105 \mathrm{~m}$. We can see that the effect of the wide $z$ filter significantly blurs the image. While this filter will ensure that all of the object will come into focus as part of the summation, it will also ensure that each part will go significantly out of focus as well and the out of focus contribution to the final summation is much greater. The result of this filter is a blurred image. However this problem can be overcome adapting the Gaussian filter. We redefine the filter with a delay in the $z$ direction with a delay parameter $f(x, y)$ that is a function of $x$ and $y$.

$$
G(x, y, z)=A \exp \left[-\left(\frac{x^{2}}{2 \sigma_{x}^{2}}+\frac{y^{2}}{2 \sigma_{y}^{2}}+\frac{(z-f(x, y))^{2}}{2 \sigma_{z}^{2}}\right)\right]
$$

with normalization factor $A$ defined as before.

This delay parameter $f(x, y)$ is given by the distance at which that part of the image at location $(x, y)$ approximately comes into focus. This can be implemented using the algorithm presented in Ref [6]. The intensity of the reconstructed image is calculated over a series of distances and the variance of the image is calculated at each location $(x, y)$ for a particular block size. This is repeated for each reconstruction distance. For a given value of $x$ and $y$ the variance is compared across the sequence of intensity reconstructions. When the variance is at a maximum we take the corresponding distance to be the in focus distance for that part of the image and we set $f(x, y)$ equal to that value. In our case we use a block size of $400 \times 400$ pixels. The depth map calculated, $f(x, y)$, is shown in Fig. $12(\mathrm{~b})$. Applying the standard 3D Gaussian filter, defined above in Eq. 15, using $z_{0}$ equal to the middle distance $0.105 \mathrm{~m}$ and using $\sigma_{x}=S_{x}, \sigma_{y}=S_{y}$ and $\sigma_{z}=7 S_{z}$ we obtain the speckle reduced image shown in Fig. 12 (a). We can see that this image is out of focus and of poor quality. However using the improved filter defined in Eq. 30 with the variable delay with $\sigma_{x}=S_{x}, \sigma_{y}=S_{y}$ and $\sigma_{z}=S_{z}$ we obtain the considerably improved image shown in Fig. 12 (c). Further speckle reduction can be achieved at the expense of some blurring by increasing the filter size. In Fig. $12(\mathrm{~d})$ we show the result for $\sigma_{x}=2 S_{x}, \sigma_{y}=2 S_{y}$ and $\sigma_{z}=2 S_{z}$. Comparing these latter two results with Fig. 12 (a) demonstrates that the filter with the variable delay affords a much improved image resulting from us being able to employ a more controlled and narrower filter in the $z$ direction. We note that the improved filter employing the variable depth parameter is not without disadvantage. It necessitates the calculation of a depth map $f(x, y)$ as described above, which requires the calculation of 


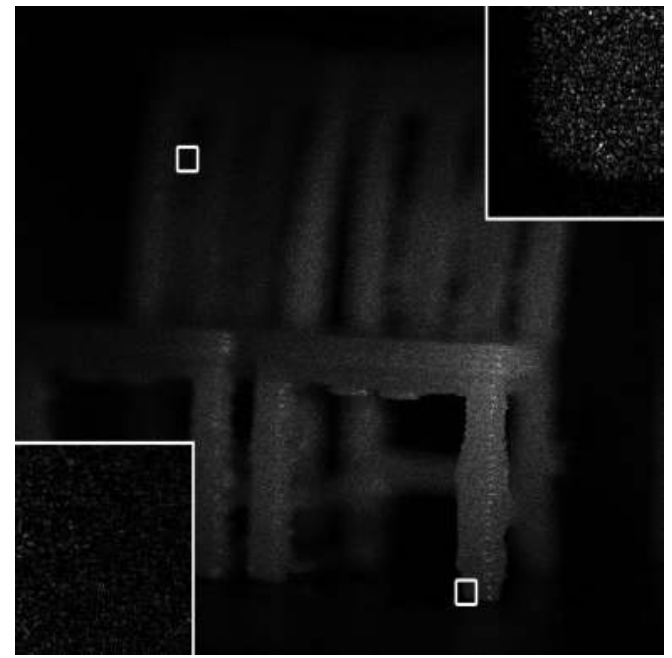

(a)

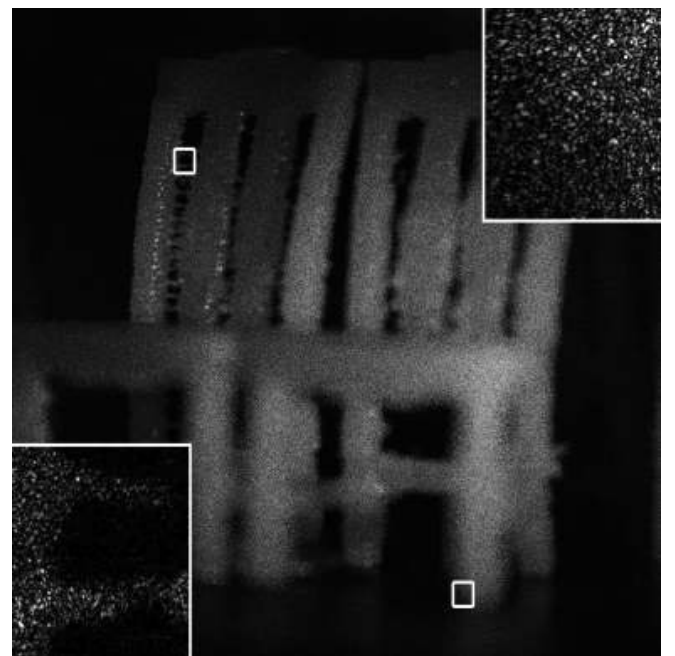

(b)

Figure 11: Reconstructions of a DH of two small chairs with (a) front in focus, and (b) back in focus. In both images two areas are highlighted and enlarged on the chairs to emphasise that in each image either the front or the back is in focus. Neither image has had any filtering applied.

multiple reconstruction depths prior to even beginning the filtering process. This can be time intensive and for the two results shown in Fig. 12 (a), the overall process required approximately two hours on a modern computer processor.

\subsection{Comparison with other techniques}

Here we present results of a comparison between the best performing filter from this paper, the 2D filter and other standard speckle reduction techniques including the mean and median filters, the discrete Fourier filter (DFF) [41] and the best performing wavelet from a previous paper of ours [42]. Table 5 provides a comprehensive set of results and reveals that 2D filtering has outperformed the other techniques, achieving a similar level of speckle reduction but maintaining a higher resolution. We have made bold the results that provide a reduction in speckle index to $\approx 0.5$ to highlight the superiority of $2 \mathrm{D}$ filtering over the other tested techniques. The next best performing method is the DFF, followed by the mean, median filters and finally the Haar mother wavelet filter. 


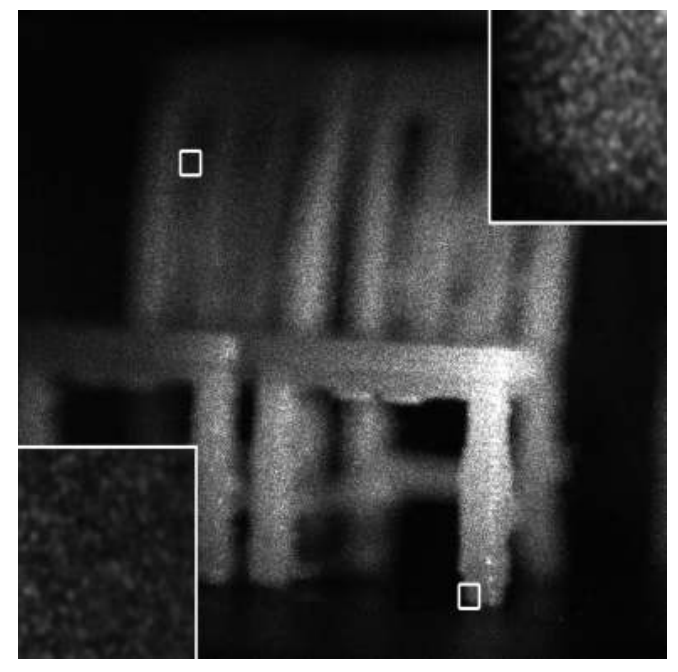

(a)

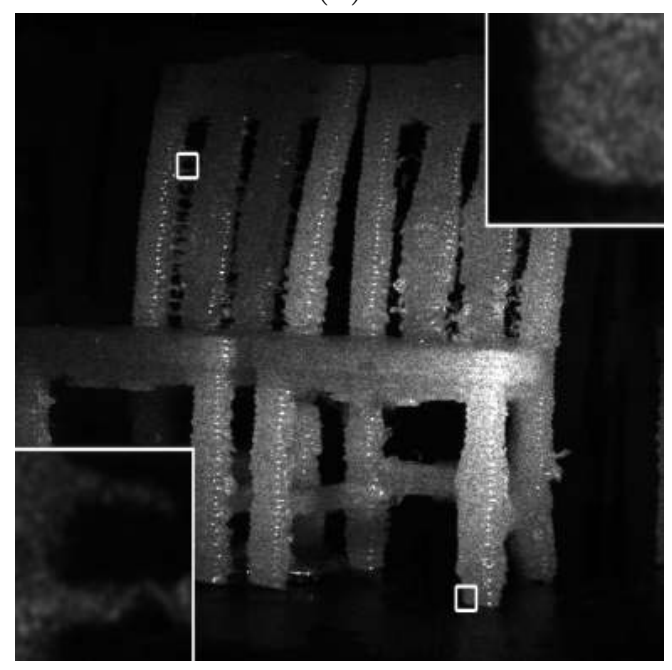

(c)

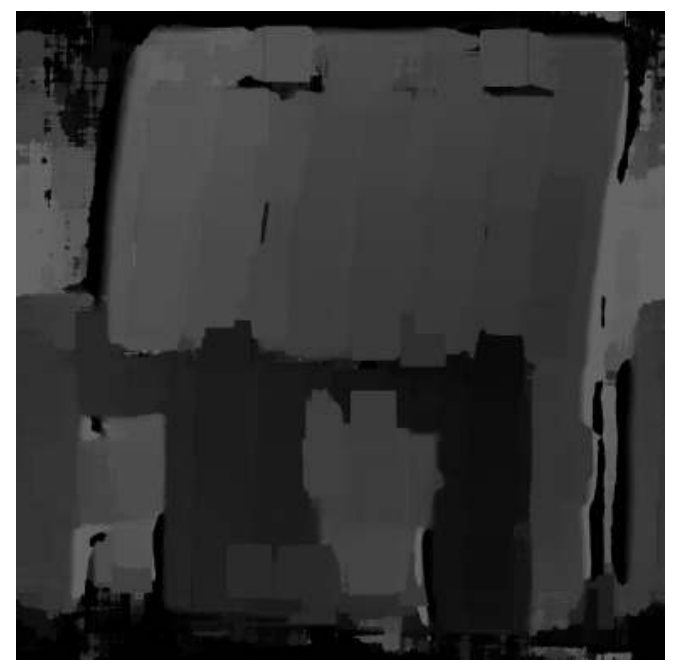

(b)

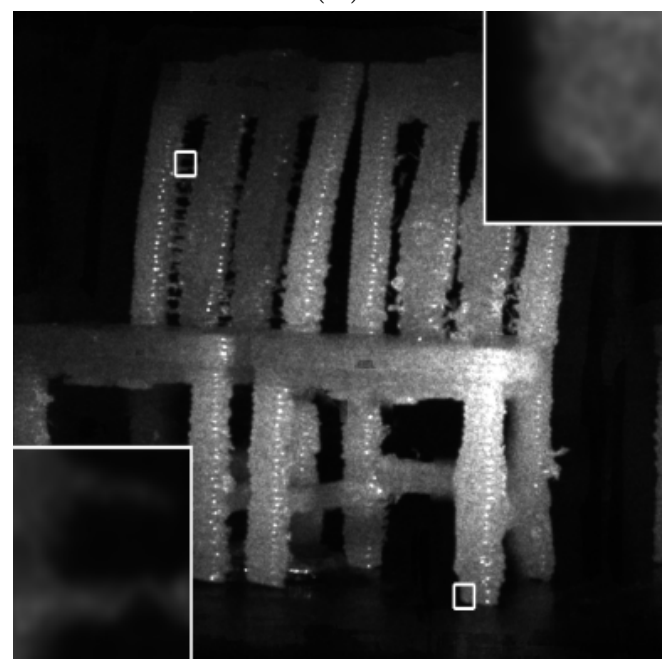

(d)

Figure 12: (a) Result of filter with size $\sigma_{x}=S_{x}, \sigma_{y}=S_{y}$ and $\sigma_{z}=7 S_{z}$ using Eq.16 with $z_{0}$ set to the middle distance $0.105 \mathrm{~m}$, (b) the depth map, $f(x, y)$ [6], (c) filter of size $\sigma_{x}=S_{x}, \sigma_{y}=S_{y}$ and $\sigma_{z}=S_{z}$, using the delay in $z$ described in Eq. 30 and (d) filter size of $\sigma_{x}=2 S_{x}, \sigma_{y}=2 S_{y}$ and $\sigma_{z}=2 S_{z}$, also using the delay in $z$ from Eq. 30 . 


\begin{tabular}{|l|c|c|c|}
\hline Technique & C & RES $x$ & RES $y$ \\
\hline 2D filtering $\left(\sigma_{\mathbf{x}}=\frac{\mathbf{S}_{\mathbf{x}}}{\mathbf{2}}, \sigma_{\mathbf{y}}=\frac{\mathbf{S}_{\mathbf{y}}}{\mathbf{2}}\right)$ & $\mathbf{0 . 5 2}$ & $\mathbf{3 5 . 9 2}$ & $\mathbf{2 8 . 5 1}$ \\
\hline 2D filtering $\left(\sigma_{x}=0.75 S_{x}, \sigma_{y}=0.75 S_{y}\right)$ & 0.37 & 32 & 22.62 \\
\hline 2D filtering $\left(\sigma_{x}=S_{x}, \sigma_{y}=S_{y}\right)$ & 0.3 & 22.62 & 17.96 \\
\hline 2D filtering $\left(\sigma_{x}=1.25 S_{x}, \sigma_{y}=1.25 S_{y}\right)$ & 0.24 & 20.16 & 16 \\
\hline DFF $(\mathbf{7 6 8} \times \mathbf{7 6 8})$ & $\mathbf{0 . 5 2}$ & $\mathbf{2 8 . 5 1}$ & $\mathbf{2 5 . 4}$ \\
\hline DFF $(512 \times 512)$ & 0.42 & 25.4 & 20.15 \\
\hline DFF $(256 \times 256)$ & 0.23 & 17.95 & 16 \\
\hline DFF $(128 \times 128)$ & 0.14 & 8.97 & 8.97 \\
\hline Haar $($ detail level 1$)$ & 0.66 & 35.92 & 25.4 \\
\hline Haar $($ detail level $\mathbf{2})$ & $\mathbf{0 . 5 3}$ & $\mathbf{2 5 . 4}$ & $\mathbf{2 2 . 6 2}$ \\
\hline Haar $($ detail level 3$)$ & 0.47 & 20.15 & 16 \\
\hline Haar $($ detail level 4$)$ & 0.45 & 16 & 14.25 \\
\hline Mean filter $(\mathbf{3} \times \mathbf{3})$ & $\mathbf{0 . 4 7}$ & $\mathbf{2 5 . 4}$ & $\mathbf{2 2 . 6 2}$ \\
\hline Mean filter $(5 \times 5)$ & 0.39 & 22.62 & 17.95 \\
\hline Mean filter $(7 \times 7)$ & 0.29 & 20.15 & 16 \\
\hline Mean filter $(9 \times 9)$ & 0.23 & 17.95 & 14.25 \\
\hline Median filter $(\mathbf{3} \times \mathbf{3})$ & $\mathbf{0 . 5 4}$ & $\mathbf{2 5 . 4}$ & $\mathbf{2 2 . 6 2}$ \\
\hline Median filter $(5 \times 5)$ & 0.46 & 22.62 & 20.15 \\
\hline Median filter $(7 \times 7)$ & 0.35 & 20.15 & 17.95 \\
\hline Median filter $(9 \times 9)$ & 0.29 & 17.95 & 16 \\
\hline
\end{tabular}

Table 5: Comparison of 2D filtering versus various speckle reduction techniques. C: speckle index, RES $x$ : resolution in the $x$ direction, RES $y$ : resolution in the $y$ direction. 


\section{Summary}

In this paper, we have reported on a Gaussian filtering technique to reduce speckle in digital holograms. Our main contribution was to use the inherent 3D nature of DHs to construct suitable 3D filters to reduce the speckle content. Using a convolution approach, a large set of quantitative results were generated for the $1 \mathrm{D}, 2 \mathrm{D}$ and $3 \mathrm{D}$ cases to show the effectiveness of the technique. We demonstrated that a reduction of the speckle content could be attained, while at the same time resolution improved in directions orthogonal to the filtering direction. We also provided qualitative evidence that 3D filtering can have a positive impact on the depth of focus of the resulting filtered image. In the following, we provide more details of the main findings in this work.

The model developed was shown to predict the reduction in speckle contrast well in the $x$ and $y$ directions, but performed less well for the $z$ direction case. This is primarily because tuning the model parameters were optimized on data taken from 2D filtering. Nonetheless, the general trend of the reduction in speckle contrast is also approximated well for filtering in $z$.

We also discussed the expected theoretical maximum recoverable frequencies and how these values relate the observable line pairs / $\mathrm{mm}$ in the image. We pointed to the fact that the theoretical values are upper limits as there are at least three factors that further reduce the bandwidth of the system; (i) the averaging effect of the pixel, which for a Fresnel system amounts to an averaging of the complex reconstruction; (ii) the effect of sampling which can result in overlapping replicas of the reconstruction and (iii) the pixel quantization, which can lead to errors in the reconstructed image [40]. Furthermore, we postulated that the speckle noise could further impact on resolution and showed that by reducing speckle noise it was possible to increase the resolution of the image.

We discussed the shape and orientation of the speckle in the 3D intensity field and postulated that the spectral method averages along lines that cut through the speckle grains. This suggests that the effective speckle size in the $z$ direction is smaller when using the spectral method to reconstruct over when the direct method is used. A numerical calculation of the speckle size confirms this. However, using the spectral method to filter in the $z$ direction has the disadvantage that reconstructions at each subsequent plane are not aligned as the field is expanding. This of course can have a negative effect on the resulting resolution. 
When filtering in the $x$ direction only, resolution in the $y$ direction initially improved (over a non-filtered reconstruction) and then after a slight degradation was maintained, all the while resolution in the $x$ direction decreased steadily with increasing kernel size. Filtering in the $y$ direction produced similar effects, with an observed slight improvement in $x$ resolution, followed by a slight degradation and leveling off, but this time with some further resolution loss. This was due to the design of the resolution chart itself, which allows for the blurring of the vertical resolution bars into each other and was not a discovery that warranted further investigation. 2D filtering in $x$ and $y$ directions resulted in a slight improvement in $x$ resolution and no change to the $y$ resolution, before a steady decrease in resolution for both $x$ and $y$. There was no initial improvement in resolution in either direction for 3D filtering and as the kernel size was increased resolution decreased steadily in both directions. As expected, in terms of resolution and speckle index, 2D filtering outperformed both 1D and 3D filtering when the criteria was to maintain resolution in both the $x$ and $y$ directions and achieve a certain level of speckle reduction. This is because the test object was planar and it can be reasonably asserted that the results might favour 3D filtering if the test object were to have an appreciable extent in the $z$ direction.

Finally, we provided qualitative evidence that 3D filtering can have a positive impact on the depth of focus of the resulting filtered image. Using a DH of two small chairs with extent in the $z$ direction, we showed that both the front and the back of the chairs could be in focus more after filtering, with the added benefit of a reduced speckle contrast, than when no filtering is applied.

\section{Acknowledgements}

This publication has emanated in part from research conducted with the financial support of Science Foundation Ireland (SFI) under Grant Number 11/SIRG/I2140. This research has also received funding in part from the European Community's Seventh Framework Programme FP7/2007 2013 under grant agreement 216105, Real 3D.

\section{References}

[1] B. R. Brown, A. W. Lohmann, Complex spatial filtering with binary masks, Applied Optics 5 (6) (1966) 967-969. 
[2] J. W. Goodman, R. W. Lawerence, Digital image formation from electronically detected holograms, Applied Physics Letters 11 (1967) 777778.

[3] U. Schnars, W. P. O. Jüptner, Direct recording of holograms by a ced target and numerical reconstruction, Applied Optics 33 (1994) 179-181.

[4] J. W. Goodman, Speckle Phenomena: Theory and Applications, Roberts \& Company, Englewood, Colorado, 2006.

[5] T. Kreis, Handbook of Holographic interferometry, Optical and Digital Methods, Wiley-VCH GmbH \& Co. KGaA, Weinheim, 2005.

[6] C. P. M. Elhinney, B. M. H. andThomas J. Naughton, Extended focused imaging for digital holograms of macroscopic three-dimensional objects, Applied Optics 47 (19) (2008) D71-9.

[7] C. P. M. Elhinney, J. B. McDonald, A. Castro, Y. Frauel, B. Javidi, T. J. Naughton, Depth-independent segmentation of macroscopic threedimensional objects encoded in single perspectives of digital holograms, Optics Letters 32 (10) (2007) 1229-1231.

[8] L. Ma, H. Wang, Y. Li, H. Jin, Numerical reconstruction of digital holograms for three-dimensional shape measurement, Journal of optics A: Pure and applied optics 6 (2004) 396-400.

[9] M. Liebling, M. Unser, Autofocus for digital Fresnel holograms by use of a fresnelet-sparsity criterion, J. Opt. Soc. Am. A 21 (12) (2004) 24242430 .

[10] J. Gillespie, R. A. King, The use of self-entropy as a focus measure in digital holography, Pattern Recognition Letters 9 (1) (1989) 19-25.

[11] B. Javidi, E. Tajahuerce, Three-dimensional object recognition by use of digital holography, Optics Letters 25 (9) (2000) 610-612.

[12] Y. Frauel, E. Tajahuerce, M. A. Castro, B. Javidi, Distortion-tolerant three-dimensional object recognition with digital holography, Applied Optics 40 (2001) 3887-3893.

[13] B. Javidi, D. Kim, Three-dimensional-object recognition by use of singleexposure on-axis digital holography, Optics Letters 30 (2005) 236-238. 
[14] D. Kim, B. Javidi, Distortion-tolerant 3-d object recognition by using single exposure on-axis digital holography, Optics Express 12 (22) (2004) $5539-5548$.

[15] F. Dubois, M.-L. N. Requena, C. Minetti, O. Monnom, E. Istasse, Partial spatial coherence effects in digital holographic microscopy with a laser source, Applied Optics 43 (5) (2004) 1131-1139.

[16] D. Kim, Reduction of coherent artifacts in dynamic holographic threedimensional displays by diffraction-specific pseudorandom diffusion, Optics Letters 29 (6) (2004) 611-613.

[17] J. Garcia-Sucerquia, J. H. Ramírez, R. Castaneda, Incoherent recovering of the spatial resolution in digital holography, Optics Communications 260 (2006) 62-67.

[18] T. Baumbach, E. Kolenović, V. Kebbel, W. Jüptner, Improvement of accuracy in digital holography by use of multiple holograms, Applied Optics 45 (24) (2006) 6077-6085.

[19] T. Nomura, M. Okamura, E. Nitanai, T. Numata, Image quality improvement of digital holography by superposition of reconstructed images obtained by multiple wavelengths, Applied Optics 47 (19) (2008) D38-D43.

[20] F. L. Clerc, M. Gross, L. Collot, Synthetic aperture experiment in the visible with on-axis digital heterodyne holography, Optics Letters 26 (2001) 1550-1552.

[21] J. H. Massig, Digital off-axis holography with a synthetic aperture, Optics Letters 27 (2002) 2179-2181.

[22] R. Binet, J. Colineau, J.-C. Lehureau, Short-range synthetic aperture at $633 \mathrm{~nm}$ by digital holography, Applied Optics 41 (2002) 4775-4782.

[23] T. Kreis, M. Adams, W. Jüptner, Aperture synthesis in digital holography, Proc. SPIE 4777 (2002) 69-76.

[24] B. M. Hennelly, T. J. Naughton, J. McDonald, Digital holographic superresolution by rotating the object wavefield, Adaptive Optics: 
Analysis and Methods/Computational Optical Sensing and Imaging/Information Photonics/Signal Recovery and Synthesis Topical Meetings on CD-ROM, OSA Technical Digest (CD), Article ID DTuD5 (2007).

[25] L. Martínez-León, B. Javidi, Synthetic aperture single-exposure on-axis digital holography, Optics Express 16 (1) (2008) 161-169.

[26] T. J. Naughton, J. B. McDonald, B. Javidi, Efficient compression of Fresnel fields for Internet transmission of three-dimensional images, Applied Optics 42 (23) (2003) 4758-4764.

[27] J. Garcia-Sucerquia, J. H. Ramírez, D. V. Prieto, Reduction of speckle noise in digital holography by using digital image processing, Optik 116 (2005) 44-48.

[28] N. Bertaux, Y. Frauel, P. Réfrégier, B. Javidi, Speckle removal using a maximum-likelihood technique with isoline gray-level regularization, Optics Society of America 21 (12) (2004) 2283-2291.

[29] F. Charrière, N. Pavillon, T. Colomb, C. Depeursinge, T. J. Hegera, E. A. D. Mitchell, P. Marquet, B. Rappaz, Living specimen tomography by digital holographic microscopy: morphometry of testate amoeba, Optics Express 14 (2006) 7005-7013.

[30] M. A. M. Gama, Speckle reduction by unidirectional averaging, Optica Acta 22 (1975) 725-730.

[31] F. Pan, W. Xiao, S. Liu, F. Wang, L. Rong, R. Li, Coherent noise reduction in digital holographic phase contrast microscopy by slightly shifting object, Optics Express 19 (5) (2011) 3862-3869.

[32] W. Xiao, J. Zhang, L. Rong, F. Pan, S. Liu, F. Wang, A. He, Improvement of speckle noise suppression in digital holography by rotating linear polarization state, Chinese Optics Letters 9 (6) (2011) 060901-060903.

[33] D. Mas, J. Garcia, C. Ferreira, L. M. Bernardo, F. Marinho, Fast algorithms for free-space diffraction patterns calculation, Optics Communications 164 (4-6) (1999) 233-245. 
[34] D. Mendlovic, Z. Zalevsky, N. Konforti, Computation considerations and fast algorithms for calculating the diffraction integral, Journal of Modern Optics 44 (2) (1997) 407-414.

[35] B. M. Hennelly, D. P. Kelly, D. S. Monaghan, N. Pandey, Zoom algorithms for digital holography, in: T. Fournel, B. Javidi (Eds.), Information Optics and photonics: Algorithms, Systems, amd Applications, Springer, 2010, pp. 187-204.

[36] L. Leushacke, M. Kirchner, Three-dimensional correlation coefficient of speckle intensity for rectangular and circular apertures, J. Opt. Soc. Am. A 7 (5) (1990) 827-832.

[37] P. Ferraro, S. D. Nicola, G. Coppola, A. Finizio, D. Alfieri, G. Pierattin, Controlling image size as a function of distance and wavelength in fresnel-transform reconstruction of digital holograms, Optics Letters 29 (2004) 854-856.

[38] R. C. Gonzalez, R. E. Woods, Digital Image Processing, Prentice-Hall, Englewood Cliffs, NJ, 2002.

[39] D. P. Kelly, B. M. Hennelly, N. Pandey, T. J. Naughton, W. T. Rhodes, Resolution limits in practical digital holographic systems, Optical Engineering 48 (9) (2009) 095801.

[40] N. Pandey, B. M. Hennelly, Quantization noise and its reduction in lensless Fourier digital holography, Applied Optics 50 (7) (2011) B58B70.

[41] J. Maycock, B. M. Hennelly, J. B. McDonald, Y. Frauel, A. Castro, B. Javidi, T. J. Naughton, Reduction of speckle in digital holography by discrete Fourier filtering, J. Opt. Soc. Am. A 24 (6) (2007) 1617-1622.

[42] K. M. Molony, J. Maycock, J. B. McDonald, B. M. Hennelly, T. J. Naughton, A comparison of wavelet analysis techniques in digital holograms, Proc. SPIE 6994 (2008) 699412. 